\title{
Enriched beam finite element models with torsion and shear warping for the analysis of thin-walled structures
}

\author{
Daniela Addessi, Paolo Di Re*, Gabriele Cimarello \\ ${ }^{a}$ Department of Structural and Geotechnical Engineering, Sapienza University of Rome, Via Eudossiana 18, 00184 Rome, Italy, \\ daniela.addessi@uniroma1.it,paolo.dire@uniromal.it,cimarello.gabriele@gmail.com
}

\section{Abstract}

This paper presents three beam Finite Element (FE) formulations developed for the analysis of thin-walled structures. These account for out-of-plane cross-section warping by removing the classical rigid body cross-section hypothesis and capture the interaction of axial/bending stress components with shear and torsion.

The beam FE models rely on different kinematic assumptions to describe out-of-plane cross-section deformations. Indeed, warping displacement field is interpolated in the element volume according to different approaches, with increasing level of accuracy and detail. First two models adopt a coarse warping description, where warping displacement field is defined as the linear combination of assumed warping profiles and unknown kinematic parameters. In the first model, these are considered as equal to the generalized cross-section torsional curvature and shear strains and a classical displacement-based formulation is adopted to derive the element governing equations. In the second model, warping parameters are assumed as independent kinematic quantities and a mixed approach is considered to derive the FE formulation. Third model, also relying on a mixed formulation, independently interpolates warping introducing additional degrees of freedom on the cross-section plane, thus, resulting in a richer description of the outof-plane deformations. This latter is also adopted to propose a numerical procedure for the warping profile evaluation of thin-walled beams subjected to torsional and shear forces, for general cross-section geometry.

The efficiency and accuracy of the proposed FE formulations are validated by simulating the response of thinwalled structures under torsion and coupled torsion/shear actions and the influence of the kinematic assumptions characterizing each formulation is discussed.

Keywords: Thin-walled beam, Warping, Finite Element, Displacement-based formulation, Mixed formulation

\section{Introduction}

Thin-walled beams are largely used in civil and mechanical engineering applications, because of their high strength against shear/bending actions compared to significant slenderness and low weight. Indeed, thin-walled elements are often adopted in construction of tall buildings, large structures and bridges, as these permit more flexibility for the

*Corresponding author:

E-mail address: paolo.dire@uniroma1.it (Paolo Di Re) 
definition of the structure geometry and guaranty higher strength and safety levels. However, in spite of the constant improvement in analysis software technology, simulation of the mechanical response of thin-walled beams is still a challenging task, as complex deformation phenomena are usually involved, even under simple static loadings.

A standard approach to analyze these structures is the adoption of beam models, which are often preferred to more demanding two-dimensional (2D) plate/shell or three-dimensional (3D) approaches, because of their efficiency and low computational cost. However, classical assumption of rigid plane cross-sections is often not suitable for thinwalled elements, as cross-sections warp in and out of their plane, when subjected to shear and torsional forces. Thus, complex stress/strain distributions arise in the structure, which are not accounted for by classical Euler-Bernoulli and Timoshenko beam theories. Moreover, coupling between axial/bending and shear/torsional stress components strongly affects the structural behavior $[1,2,3]$.

Over past years, many works focused on the analysis of thin-walled beams and studied the influence of crosssection warping effects on structural response $[4,5,6,7,8]$. Starting from the first proposals by Vlasov [9] and Benscoter [10], several enhanced beam theories and numerical models were presented, often relying on Finite Element (FE) procedures $[11,12,13,14]$. Vlasov's theory includes cross-section warping due to torsion and is based on the assumption that in-plane cross-section distorsions are negligible. Due to the small thickness of the membratures, this considers out-of-plane warping as uniform across web and flanges of the element and, thus, assumes out-of-plane axial displacements as proportional to the element torsional curvature. These conditions usually hold true for doublysymmetric open cross sections, where shear strains vanish at cross-section mid-surface, but are not met for closed and/or non-symmetric profiles [15]. More general assumptions are made in Benscoter's theory, where, keeping all the other hypotheses valid, out-of-plane displacements are assumed as proportional to an independent kinematic warping parameter, which is found to be function of the cross-section torsional rotation.

First proposals were devoted to the analysis of slender thin-walled elements [16]. Hence, by neglecting beam shear flexibility, warping theories were combined with Euler-Bernoulli beam formulation, to account for coupling between axial stresses due to bending and torsion. A comprehensive review of beam models accounting for cross-section warping proposed in the last decades of previous century and mainly based on aforementioned theories is reported in [17]. Among them, it is worth mentioning those in $[18,19]$, which combine Timoshenko's and Vlasov's theories to account for both torsional warping and shear flexibility, and works by Shakourzadeh et al. [20] and by Saade et al. [21], which present Benscoter-based models and provide detailed discussions on the applicability of Vlasov's theory. In the last decades, enriched models were proposed to include also cross-section warping due to shear forces, e.g. $[22,23,24,25,26]$, proving that formulations able to account for these deformation modes provide significantly more reliable and accurate results in the analysis of non-symmetric cross-section elements and/or buckling phenomena $[27,28]$.

More sophisticated formulations were recently presented in [29, 30, 31, 32], where cross-section out-of-plane displacements are described through the composition of independent warping modes, evaluated by imposing equilibrium conditions and used as interpolation shape functions. Genoese et al. [33, 34] defined a mixed Hellinger-Reissner 
beam model based on a stress field description that results as the sum of the exact de Saint Venant contribution and some further terms due to variable warping.

This paper presents three 3D beam FE formulations developed for the analysis of thin-walled structures. Starting from Timoshenko beam theory and assuming small displacements and strains, enriched kinematic descriptions are introduced, so that beam cross-sections can undergo out-of-plane deformations, yet remaining rigid in their plane. Warping effects are assumed as related to both torsion and shear and linked to specific kinematic quantities that vary along the element axis, so that the influence of warping restraints at the element boundaries and resulting shear-lag phenomena are properly account for.

First model is based on Vlasov's theory, which is here enriched to also include shear warping. Thus, the out-ofplane warping displacement field is introduced in addition to those resulting from the cross-section rigid motions. This is defined as the linear combination of three warping functions, a priori defined over the element cross-section, and unknown generalized cross-section kinematic parameters. These are assumed as equal to the generalized cross-section torsional curvature and shear strains. A displacement-based approach is adopted to derive the element governing equations, that is generalized cross-section displacements are interpolated along the element axis by means of shape functions. To this end, additional degrees of freedom (DOFs) are introduced at the two element end nodes, corresponding to torsional curvature and shear strains attained at the element end cross-sections. These are used for two purposes. On the one hand, the additional DOFs are used to obtain parabolic and linear interpolation along the element axis of the cross-section torsional curvature and shear strains, respectively, and, thus, to have similar interpolation order for the corresponding warping modes. On the other hand, shear strain DOFs are used to perform an enhanced interpolation of bending/shear quantities [35] that prevent shear-locking issues occurring in the standard two-node displacement-based models with linear interpolation of the cross-section transversal displacements and rotations [36, 37, 38, 39].

Second model is based on Benscoter's warping theory, also enriched to include shear warping. Hence, similarly to the first model, cross-section out-of-plane displacement field is described by the linear combination of three a priori defined warping functions and corresponding generalized cross-section parameters for torsion and shear, but these latter are assumed as independent kinematic quantities. Hence, better warping description than first model is considered. Warping displacement interpolation is performed by introducing three DOFs at the element end nodes and at a variable number of internal nodes located along the axis. Moreover, as force-based and mixed formulations are free from shear-locking and have higher performances than displacement-based FEs [40, 41], as opposed to the first model, a mixed approach is used in this case. Together with the additional warping field, generalized cross-section stresses are interpolated along the element axis according to equilibrium conditions. The governing equations are, thus, derived by imposing the stationarity of an extended four-field $\mathrm{Hu}$-Washizu variational functional.

Third model is the general extension of second formulation. This was proposed by the authors in previous works and applied to the analysis of damaging structures subjected to shear and torsional forces [42, 43, 44] and thinwalled structures under dynamic loadings [45, 46, 47]. A mixed FE approach is used to derive the element governing equations, but a more general and accurate warping description is considered. Indeed, a variable number of additional 


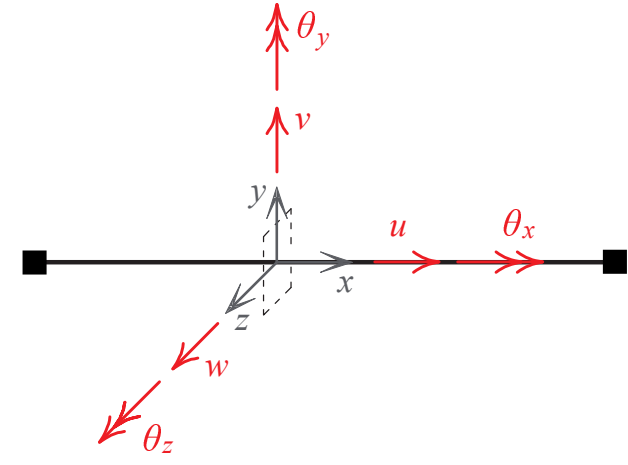

(a)

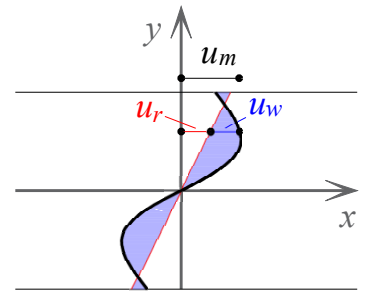

(b)

Figure 1: Cross-section (a) generalized displacement components and (b) out-of-plane warping displacement lying on the cross-section plane and constituting an orthogonal triad (Fig. 1(a)).

DOFs is introduced at each controlling node and the additional warping displacement field is interpolated at two levels: along the beam axis and over the cross-section plane. Hence, higher order descriptions are allowed for the out-of-plane deformations and complete coupling of all stress components is ensured.

To evaluate the warping functions required in the first two formulations a numerical procedure is proposed. By adopting the third mixed FE formulation and enforcing uniform warping conditions, linear elastic solutions are determined for a beam subjected to uniform torsional curvature and shear strains. The corresponding cross-section warping profiles are, thus, computed and assumed as warping functions.

Numerical simulations are, finally, conducted to test and compare the performances of the three proposed FE models. The response of four thin-walled specimens subjected to torsional and shear loads is studied, considering various cross-section shapes to investigate the applicability of Vlasov's and Benscoter's assumptions. Convergence studies are performed to highlight the influence on the structural response of the assumed warping interpolation order along the element axis and over the cross-section.

\section{General assumptions}

An enriched 3D beam formulation is considered including out-of-plane deformations for the cross-section to describe warping phenomena, while this remains rigid in its plane. Small displacement and strain hypothesis is assumed. The beam domain is composed by the $1 \mathrm{D}$ locus of cross-section centroids, $\{x \in[0, L]\}$, and the $2 \mathrm{D}$ domain of the cross-section $A(x)$. The local intrinsic reference system $(x, y, z)$ is formed by axis $x$ and the two axes $y$ and $z$

$$
\mathbf{u}_{m}(x, y, z)=\mathbf{u}_{r}(x, y, z)+\mathbf{u}_{w}(x, y, z)
$$

where $\mathbf{u}_{r}(x, y, z)=\left\{u_{r}(x, y, z) \quad v_{r}(x, y, z) \quad w_{r}(x, y, z)\right\}^{T}$ defines the cross-section rigid body motions, and $\mathbf{u}_{w}(x, y, z)=$ $\left\{u_{w}(x, y, z) \quad 0 \quad 00\right\}^{T}$, the out-of-plane displacement due to warping. Rigid displacements are expressed in terms of 
generalized cross-section displacements as $\mathbf{u}_{r}(x, y, z)=\alpha(y, z) \mathbf{u}_{s}(x)$, where $\mathbf{u}_{s}(x)$ is the vector collecting the crosssection translations, $u(x), v(x), w(x)$, and rotations, $\theta_{x}(x), \theta_{y}(x), \theta_{z}(x)$, (Fig. 1(a)), i.e.:

$$
\mathbf{u}_{s}(x)=\left\{\begin{array}{llllll}
u(x) & \theta_{z}(x) & v(x) & \theta_{x}(x) & \theta_{y}(x) & w(x)
\end{array}\right\}^{T},
$$

and matrix $\alpha(y, z)$ is the compatibility operator, defining the rigid motions as:

$$
\alpha(y, z)=\left[\begin{array}{cccccc}
1 & -y & 0 & 0 & z & 0 \\
0 & 0 & 1 & -z & 0 & 0 \\
0 & 0 & 0 & y & 0 & 1
\end{array}\right] .
$$

As the cross-section in-plane deformations are neglected, displacement field $u_{w}(x, y, z)$ is the only non-zero component of vector $\mathbf{u}_{w}(x, y, z)$, representing the out-of-plane warping displacement of point $m$. This is written as the product of warping functions defined over the cross-section area, collected in the row vector $\mathbf{M}_{\eta}(y, z)$, and independent generalized cross-section kinematic quantities, defined along the element axis and collected in the column vector $\eta_{s}(x)$. The latter provides the amplitudes of the warping displacements associated to each warping function in $\mathbf{M}_{\eta}(y, z)$. Hence, it results:

$$
u_{w}(x, y, z)=\mathbf{M}_{\eta}(y, z) \eta_{s}(x)
$$

As displacements $\mathbf{u}_{r}(x, y, z)$ and $\mathbf{u}_{w}(x, y, z)$ are assumed to be orthogonal, the warping functions $\mathbf{M}_{\eta}(y, z)$ have to satisfy the following conditions $[21,24]$ :

$$
\int_{A(x)} \mathbf{M}_{\eta}(y, z) d A=0, \quad \int_{A(x)} y \mathbf{M}_{\eta}(y, z) d A=0, \quad \int_{A(x)} z \mathbf{M}_{\eta}(y, z) d A=0 .
$$

These are ensured by a proper definition of the warping functions $\mathbf{M}_{\eta}(y, z)$, as described in the following sections.

According to the classical de Saint-Venant's beam theory, three non-zero strain components are considered at point $m$, that is the axial elongation $\varepsilon_{x x}(x, y, z)$ and the two transverse shear strains between material fibers parallel to $x$ and those lying in the cross-section plane, $\gamma_{x y}(x, y, z)$ and $\gamma_{x z}(x, y, z)$. These are collected in vector $\varepsilon_{m}(x, y, z)$ and expressed

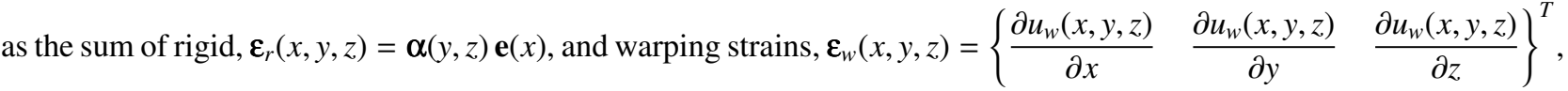
as:

$$
\boldsymbol{\varepsilon}_{m}(x, y, z)=\boldsymbol{\varepsilon}_{r}(x, y, z)+\boldsymbol{\varepsilon}_{w}(x, y, z),
$$

where vector $\mathbf{e}(x)$ contains the generalized cross-section strains, i.e. axial strain, $\varepsilon_{G}(x)$, flexural curvatures, $\chi_{z}(x)$ and $\chi_{y}(x)$, torsional curvature, $\chi_{x}(x)$, and shear strains, $\gamma_{y}(x)$ and $\gamma_{z}(x)$, and results as:

$$
\mathbf{e}(x)=\left(\begin{array}{c}
\varepsilon_{G}(x) \\
\chi_{z}(x) \\
\gamma_{y}(x) \\
\chi_{x}(x) \\
\chi_{y}(x) \\
\gamma_{z}(x)
\end{array}\right)=\left(\begin{array}{c}
u^{\prime}(x) \\
\theta_{z}^{\prime}(x) \\
v^{\prime}(x)-\theta_{z}(x) \\
\theta_{x}^{\prime}(x) \\
\theta_{y}^{\prime}(x) \\
w^{\prime}(x)+\theta_{y}(x)
\end{array}\right)=\mathbf{D}(x) \mathbf{u}_{s}(x) .
$$


The apex denotes the derivative with respect to $x$ of the variable and matrix $\mathbf{D}(x)$ is the compatibility differential operator.

The stress components work-conjugate to $\boldsymbol{\varepsilon}_{m}(x, y, z)$ are collected in the stress vector $\boldsymbol{\sigma}_{m}(x, y, z)$, containing the axial stress, $\sigma_{x x}(x, y, z)$, and the shear stresses in the plane of the cross-section, $\tau_{x y}(x, y, z)$ and $\tau_{x z}(x, y, z)$. By applying the virtual work principle, the standard beam internal forces, $\mathbf{s}(x)$, namely axial stress, $N(x)$, bending moments, $M_{z}(x)$ and $M_{y}(x)$, torsional moment, $M_{x}(x)$, and shear stresses, $T_{y}(x)$ and $T_{z}(x)$, are derived as:

$$
\mathbf{s}(x)=\int_{A(x)} \alpha^{T}(y, z) \boldsymbol{\sigma}_{m}(x, y, z) d A .
$$

Strains, $\boldsymbol{\varepsilon}_{m}(x, y, z)$, and stresses, $\boldsymbol{\sigma}_{m}(x, y, z)$ at material point $m$, are related by the material constitutive law. This is expressed in the general total form as [48, 43]:

$$
\sigma_{m}(x, y, z)=\tilde{\sigma}_{m}\left(\varepsilon_{m}(x, y, z)\right)
$$

or, in incremental form, as:

$$
\Delta \boldsymbol{\sigma}_{m}(x, y, z)=\mathbf{k}_{m}(x, y, z) \Delta \boldsymbol{\varepsilon}_{m}(x, y, z)
$$

where $\Delta$ indicates the increment of the quantity and $\mathbf{k}_{m}(x, y, z)$ is the material tangent stiffness matrix, defined as:

$$
\mathbf{k}_{m}(x, y, z)=\frac{\partial \boldsymbol{\sigma}_{m}(x, y, z)}{\partial \boldsymbol{\varepsilon}_{m}} .
$$

\section{Enhanced beam FE formulations with cross-section warping}

In the following, three different beam finite element (FE) formulations for straight beams with cross-section warping are presented. Each assumes a different representation of the warping displacement field $u_{w}(x, y, z)$, i.e. Eq. (4) is differently specified on the basis of specific kinematic assumptions. Hence, different kinematic and static quantities associated to warping are considered, as described. The three formulations are called hereafter as Enriched Vlasov Displacement Element (EVDE), Simplified Warping Mixed Element (SWME) and Enriched Warping Mixed Element (EWME), respectively. These are described referring to the element local reference system and considering the following common assumptions.

A two-node 3D beam FE is formulated and, without loss of generality, the local reference system is assumed to coincide with the beam intrinsic orthogonal system $(x, y, z)$, where axis $x$ connects the element end nodes $i$ and $j$. Six standard DOFs are considered at each end node, that is three translations, collected in vectors $\mathbf{u}_{i}$ and $\mathbf{u}_{j}$, and three rotations contained in vectors $\theta_{i}$ and $\theta_{j}$, at node $i$ and $j$ respectively. The element nodal displacement vector is, thus, written as:

$$
\mathbf{u}=\left\{\begin{array}{llll}
\mathbf{u}_{i}^{T} & \boldsymbol{\theta}_{i}^{T} & \mathbf{u}_{j}^{T} & \boldsymbol{\theta}_{j}^{T}
\end{array}\right\}^{T} .
$$




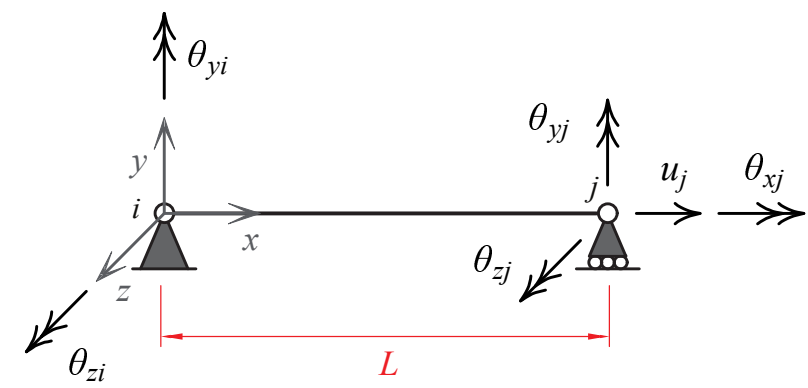

Figure 2: Basic deformation displacement DOFs in the local system $(i ; x, y, z)$ assumed as standard reference configuration

According to force-based equlibrated formulations for beams [40, 41], a reference basic configuration is defined to remove rigid body motions and derive the element governing equations, as depicted in Fig. 2. Then, the basic deformation displacement vector $\mathbf{v}$ is introduced, resulting as:

$$
\mathbf{v}=\left\{\begin{array}{llllll}
u_{j} & \theta_{z i} & \theta_{z j} & \theta_{x j} & \theta_{y i} & \theta_{y j}
\end{array}\right\}^{T}=\mathbf{a}_{v} \mathbf{u}
$$

where $\mathbf{a}_{v}$ is the kinematic operator removing the element rigid body motions, i.e.:

$$
\mathbf{a}_{v}=\left[\begin{array}{cccccccccccc}
-1 & 0 & 0 & 0 & 0 & 0 & 1 & 0 & 0 & 0 & 0 & 0 \\
0 & 1 / L & 0 & 0 & 0 & 1 & 0 & -1 / L & 0 & 0 & 0 & 0 \\
0 & 1 / L & 0 & 0 & 0 & 0 & 0 & -1 / L & 0 & 0 & 0 & 1 \\
0 & 0 & 0 & -1 & 0 & 0 & 0 & 0 & 0 & 1 & 0 & 0 \\
0 & 0 & -1 / L & 0 & 1 & 0 & 0 & 0 & 1 / L & 0 & 0 & 0 \\
0 & 0 & -1 / L & 0 & 0 & 0 & 0 & 0 & 1 / L & 0 & 1 & 0
\end{array}\right],
$$

and $L$ is the element undeformed length. The basic nodal force vector $\mathbf{q}$, work-conjugate to $\mathbf{v}$, results as:

$$
\mathbf{q}=\left\{\begin{array}{llllll}
p_{x j} & m_{z i} & m_{z j} & m_{x j} & m_{y i} & m_{y j}
\end{array}\right\}^{T} .
$$

Moreover, all proposed FE formulations include additional DOFs properly introduced to describe cross-section warping, as detailed in the following.

\subsection{Enriched Vlasov Displacement Element (EVDE) formulation}

The EVDE formulation introduces three additional DOFs at each element end node, namely $\chi_{x i}, \gamma_{y i}$ and $\gamma_{z i}$, for node $i$, and $\chi_{x j}, \gamma_{y j}$ and $\gamma_{z j}$, for node $j$ (Fig. 3). DOF $\chi_{x i / j}$ is the torsional curvature at the element end cross-section and is used to describe cross-section warping due to torsion, while $\gamma_{y i / j}, \gamma_{z i / j}$ are the cross-section shear strains used to describe warping due to shear. Hence, the nodal displacement vector results as:

$$
\hat{\mathbf{u}}=\left\{\begin{array}{ll}
\mathbf{u}^{T} & \eta^{T}
\end{array}\right\}^{T},
$$




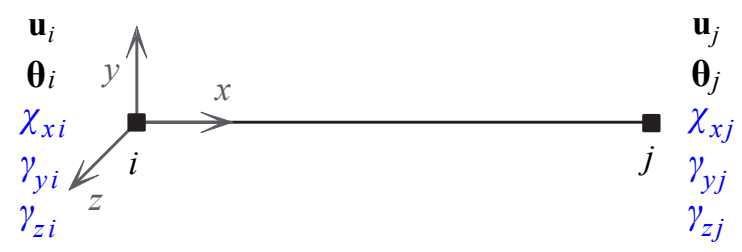

Figure 3: Standard (black) and warping (blue) nodal DOFs for the EVDE model

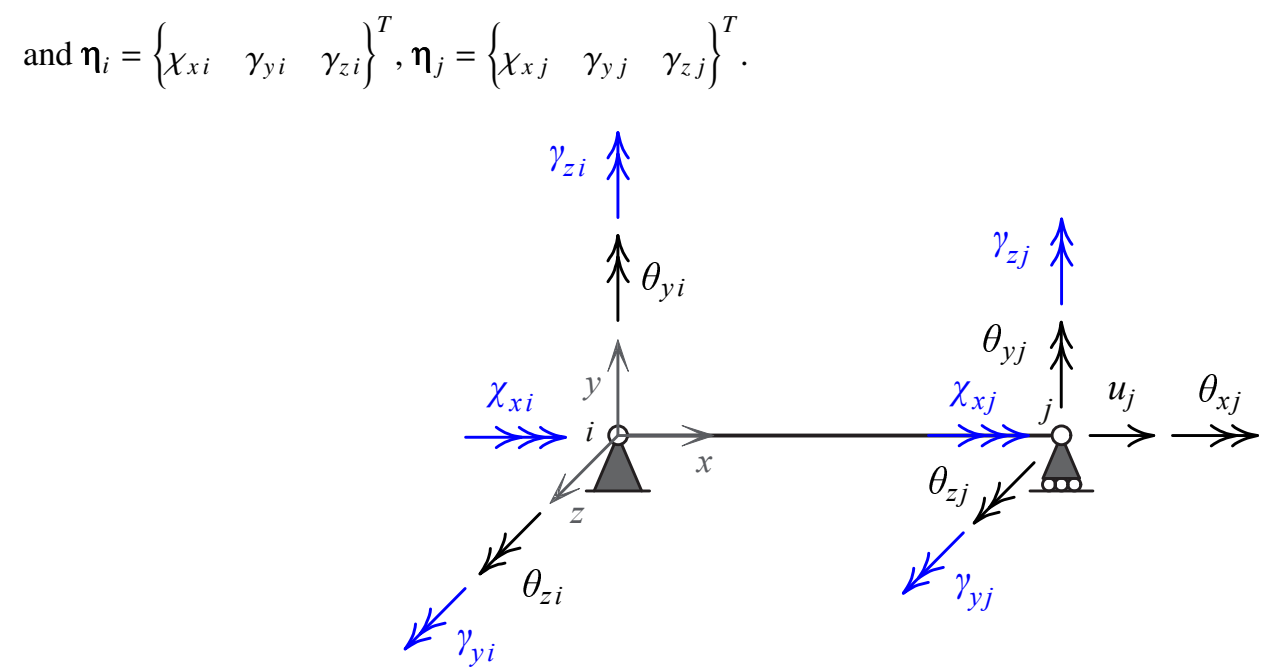

where:

$$
\eta=\left\{\eta_{i}^{T} \quad \eta_{j}^{T}\right\}^{T}
$$

Figure 4: EVDE reference configuration in the local system $(i ; x, y, z)$ with basic displacement (black) and warping (blue) DOFs

Considering the reference configuration where the element rigid body motions are eliminated, the deformation displacement and warping DOFs are collected in the extended deformation displacement vector $\hat{\mathbf{v}}$, which results as (Fig. 4):

$$
\hat{\mathbf{v}}=\left\{\begin{array}{ll}
\mathbf{v}^{T} & \eta^{T}
\end{array}\right\}^{T}=\hat{\mathbf{a}}_{v} \hat{\mathbf{u}}
$$

where matrix $\hat{\mathbf{a}}_{v}$ is defined as:

$$
\hat{\mathbf{a}}_{v}=\left[\begin{array}{ll}
\mathbf{a}_{v} & \mathbf{0} \\
\mathbf{0} & \mathbf{I}
\end{array}\right] .
$$

Matrix I extracts the warping displacements from $\hat{\mathbf{u}}$ and reorders them according to $\hat{\mathbf{v}}$. $\mathbf{0}$ denotes null matrix with proper dimensions. The internal force vector $\hat{\mathbf{q}}$, work-conjugate to $\hat{\mathbf{v}}$, results as:

$$
\hat{\mathbf{q}}=\left\{\begin{array}{ll}
\mathbf{q}^{T} & \beta^{T}
\end{array}\right\}^{T},
$$


where $\beta$ collects the bi-moments attained at the end beam cross-sections, i.e.:

$$
\beta=\left\{\begin{array}{llllll}
b_{x i} & b_{x j} & b_{y i} & b_{y j} & b_{z i} & b_{z j}
\end{array}\right\}^{T} .
$$

Vectors $\hat{\mathbf{q}}$ and $\hat{\mathbf{v}}$ are related by the element constitutive law derived in Section 3.1.3.

\subsubsection{Warping description and cross-section behavior}

The EVDE model relies on Vlasov's description of warping displacement field $u_{w}(x, y, z)$ under torsion [9], enriched to include cross-section warping due to shear [21, 24, 25]. Hence, Eq. (4) is written in the form:

$$
u_{w}(x, y, z)=M_{\eta x}(y, z) \eta_{x}(x)+M_{\eta y}(y, z) \eta_{y}(x)+M_{\eta z}(y, z) \eta_{z}(x),
$$

so that $\mathbf{M}_{\eta}(y, z)=\left\{M_{\eta x}(y, z) \quad M_{\eta y}(y, z) \quad M_{\eta z}(y, z)\right\}$ with $M_{\eta x}(y, z), M_{\eta y}(y, z)$ and $M_{\eta z}(y, z)$, being the three warping functions associated to torsion and shear along $y$ - and $z$-directions, respectively, while $\eta_{s}(x)=\left\{\begin{array}{lll}\eta_{x}(y, z) & \eta_{y}(y, z) & \eta_{z}(y, z)\end{array}\right\}^{T}$, with $\eta_{x}(x), \eta_{y}(x)$ and $\eta_{z}(x)$ being the associated warping parameters. According to Vlasov's theory, these latter are assumed as equal to the cross-section torsional curvature, $\chi_{x}(x)$, and shear strains, $\gamma_{y}(x)$ and $\gamma_{z}(x)$, respectively, that is:

$$
\eta_{x}(x)=\chi_{x}(x), \quad \eta_{y}(x)=\gamma_{y}(x), \quad \eta_{z}(x)=\gamma_{z}(x) .
$$

This assumption derives from observing that, for very thin elements, uniform warping displacements can be considered across the thickness of the cross-section membratures and, thus, transverse shear strains in this direction can be neglected $[21,20]$. This is usually adequate for open cross-sections, but can lead to incorrect solutions for closed profiles. In the proposed formulation, Vlasov's theory is enriched by assuming functions $M_{\eta x}(y, z), M_{\eta y}(y, z)$ and $M_{\eta z}(y, z)$ that allow non-uniform warping distribution across the member thickness, while Eq. (23) is adopted [25]. Indeed, warping functions $M_{\eta x}(y, z), M_{\eta y}(y, z)$ and $M_{\eta z}(y, z)$ are determined according to the numerical procedure described in Section 4.1, which leads to a more general formulation suitable for both open and closed cross-sections, as well as symmetric and non-symmetric, thin- and thick-walled cross-sections, and ensures the orthogonality conditions between rigid and warping displacements, as required by Eq. (5). Some examples of warping functions are depicted in Fig. 5 for rectangular, I-shaped, C-shaped and boxed cross-section.

The EVDE formulation is based on the independent interpolation of the two strain fields $\gamma_{y}(x)$ and $\gamma_{z}(x)$ instead of the rotation fields $\theta_{y}(x)$ and $\theta_{z}(x)$. Thus, the modified cross-section kinematic vector $\hat{\mathbf{u}}_{s}(x)$ is introduced as:

$$
\hat{\mathbf{u}}_{s}(x)=\left\{\begin{array}{llllll}
u(x) & v(x) & \theta_{x}(x) & w(x) & \gamma_{y}(x) & \gamma_{z}(x)
\end{array}\right\}^{T} .
$$




\begin{tabular}{|l|lll|}
\hline Rectangular & $M_{\eta x}(y, z)$ & $M_{\eta y}(y, z)$ & $M_{\eta z}(y, z)$ \\
\hline I-shaped & & \\
\hline C-shaped & & \\
\hline Boxed & &
\end{tabular}

Figure 5: Warping function shape for typical cross-sections related to torsion, $M_{\eta x}$, and shear, $M_{\eta y}$ and $M_{\eta_{z}}$, states.

Vector $\mathbf{u}_{s}(x)$ can be deduced from $\hat{\mathbf{u}}_{s}(x)$ by means of the following operator:

229

$$
\mathbf{a}_{u}(x)=\left[\begin{array}{cccccc}
1 & 0 & 0 & 0 & 0 & 0 \\
0 & \frac{d}{d x} & 0 & 0 & -1 & 0 \\
0 & 1 & 0 & 0 & 0 & 0 \\
0 & 0 & 1 & 0 & 0 & 0 \\
0 & 0 & 0 & -\frac{d}{d x} & 0 & 1 \\
0 & 0 & 0 & 1 & 0 & 0
\end{array}\right] .
$$

Moreover, an extended generalized cross-section strain vector is introduced, defined as:

$$
\hat{\mathbf{e}}(x)=\left\{\begin{array}{llll}
\mathbf{e}^{T}(x) & \zeta_{x}(x) & \zeta_{y}(x) & \zeta_{z}(x)
\end{array}\right\}^{T}=\hat{\mathbf{D}}(x) \hat{\mathbf{u}}_{s}(x),
$$


232 where $\zeta_{x}(x)=\chi_{x}^{\prime}(x), \zeta_{y}(x)=\gamma_{y}^{\prime}(x), \zeta_{z}(x)=\gamma_{z}^{\prime}(x)$ and $\hat{\mathbf{D}}(x)$ is the extended kinematic differential operator, resulting 233 as:

$$
\hat{\mathbf{D}}(x)=\left[\begin{array}{cccccc}
\frac{d}{d x} & 0 & 0 & 0 & 0 & 0 \\
0 & \frac{d^{2}}{d x^{2}} & 0 & 0 & -\frac{d}{d x} & 0 \\
0 & 0 & 0 & 0 & 1 & 0 \\
0 & 0 & \frac{d}{d x} & 0 & 0 & 0 \\
0 & 0 & 0 & -\frac{d^{2}}{d x^{2}} & 0 & \frac{d}{d x} \\
0 & 0 & 0 & 0 & 0 & 1 \\
0 & 0 & \frac{d^{2}}{d x^{2}} & 0 & 0 & 0 \\
0 & 0 & 0 & 0 & \frac{d}{d x} & 0 \\
0 & 0 & 0 & 0 & 0 & \frac{d}{d x}
\end{array}\right] .
$$

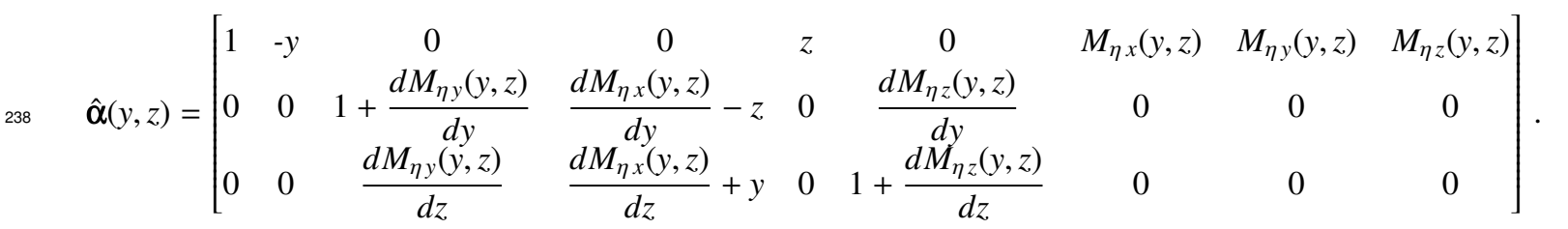

According to (6), the material strains are determined as:

$$
\boldsymbol{\varepsilon}_{m}(x, y, z)=\boldsymbol{\varepsilon}_{r}(x, y, z)+\boldsymbol{\varepsilon}_{w}(x, y, z)=\hat{\alpha}(y, z) \hat{\mathbf{e}}(x),
$$

The application of the virtual work principle, considering a virtual rigid strain as $\delta \boldsymbol{\varepsilon}_{m}(x, y, z)=\hat{\alpha}(y, z) \delta \hat{\mathbf{e}}(x)$, leads to the definition of the extended beam internal forces $\hat{\mathbf{s}}(x)$, as:

$$
\hat{\mathbf{s}}(x)=\int_{A(x)} \hat{\boldsymbol{\alpha}}^{T}(y, z) \boldsymbol{\sigma}_{m}(x, y, z) d A,
$$

with:

$$
\hat{\mathbf{s}}(x)=\left\{\begin{array}{lllllllll}
N(x) & M_{z}(x) & T_{y}^{p}(x) & M_{x}^{p}(x) & M_{y}(x) & T_{z}^{p}(x) & B_{x}(x) & B_{y}(x) & B_{z}(x)
\end{array}\right\}^{T}
$$

where quantities $B_{x}(x), B_{y}(x)$ and $B_{z}(x)$, are the additional generalized stresses work-conjugate to the generalized strains $\zeta_{x}(x), \zeta_{y}(x), \zeta_{z}(x)$ and known as bi-moments [24, 9, 33]. These are defined as:

$$
\begin{aligned}
& B_{x}(x)=\int_{A(x)} M_{\eta x}(y, z) \sigma_{x x}(x, y, z) d A, \\
& B_{y}(x)=\int_{A(x)} M_{\eta y}(y, z) \sigma_{x x}(x, y, z) d A, \\
& B_{z}(x)=\int_{A(x)} M_{\eta z}(y, z) \sigma_{x x}(x, y, z) d A,
\end{aligned}
$$


while quantities $M_{x}^{p}(x), T_{y}^{p}(x)$ and $T_{z}^{p}(x)$ are usually referred to as primary torsional moment and shear stresses.

These result as the sum of two contributions: the standard torsional moment and shear stresses, $M_{x}(x), T_{y}(x)$ and $T_{z}(x)$, associated to the generalized strains for the rigid cross-section, and additional quantities, $M_{x}^{s}(x), T_{y}^{s}(x)$ and $T_{z}^{s}(x)$, associated to the warping strains, that is:

where:

$$
\begin{aligned}
M_{x}(x) & =\int_{A(x)}\left[y \tau_{x z}(x, y, z)-z \tau_{x y}(x, y, z)\right] d A, \\
T_{y}(x) & =\int_{A(x)} \tau_{x y}(x, y, z) d A, \\
T_{z}(x) & =\int_{A(x)} \tau_{x z}(x, y, z) d A,
\end{aligned}
$$

$$
\begin{aligned}
M_{x}^{p}(x) & =M_{x}(x)+M_{x}^{s}(x), \\
T_{y}^{p}(x) & =T_{y}(x)+T_{y}^{s}(x), \\
T_{z}^{p}(x) & =T_{z}(x)+T_{z}^{s}(x),
\end{aligned}
$$

and:

$$
\begin{aligned}
M_{x}^{s}(x) & =\int_{A(x)}\left[\frac{d M_{\eta x}(y, z)}{d y} \tau_{x y}(x, y, z)+\frac{d M_{\eta x}(y, z)}{d z} \tau_{x z}(x, y, z)\right] d A, \\
T_{y}^{s}(x) & =\int_{A(x)}\left[\frac{d M_{\eta y}(y, z)}{d y} \tau_{x y}(x, y, z)+\frac{d M_{\eta y}(y, z)}{d z} \tau_{x z}(x, y, z)\right] d A, \\
T_{z}^{s}(x) & =\int_{A(x)}\left[\frac{d M_{\eta z}(y, z)}{d y} \tau_{x y}(x, y, z)+\frac{d M_{\eta z}(y, z)}{d z} \tau_{x z}(x, y, z)\right] d A .
\end{aligned}
$$

The latter are usually known as bi-shears or secondary torsional moment and shear stresses.

By differentiating $\hat{\mathbf{s}}(x)$ with respect to $\hat{\mathbf{e}}(x)$ and considering Eqs. (30), (28) and (11), the cross-section generalized tangent stiffness matrix is obtained, resulting as:

$$
\hat{\mathbf{k}}_{s}(x)=\frac{\partial \hat{\mathbf{s}}(x)}{\partial \hat{\mathbf{e}}}=\int_{A(x)} \hat{\alpha}^{T}(y, z) \mathbf{k}_{m}(x, y, z) \hat{\alpha}(y, z) d A .
$$

This governs the generalized cross-section constitutive relationship in incremental form, that is:

$$
\Delta \hat{\mathbf{S}}(x)=\hat{\mathbf{k}}_{s}(x) \Delta \hat{\mathbf{e}}(x) .
$$

\subsubsection{Cross-section displacement interpolation}

According to the displacement-based approach, the generalized cross-section displacements, $\hat{\mathbf{u}}_{s}(x)$, are interpolated along the element axis by means of shape functions, using Lagrange and Hermite polynomials. The axial displacement field, $u(x)$, and the shear strains, $\gamma_{y}(x)$ and $\gamma_{z}(x)$ are assumed as linear functions, while the transverse displacements, $v(x)$ and $w(x)$, and the torsional rotation, $\theta_{x}(x)$, are assumed as cubic. Thus, the relationship between vector $\hat{\mathbf{u}}_{s}(x)$ and the basic displacements $\hat{\mathbf{v}}$ is expressed in compact form as:

$$
\hat{\mathbf{u}}_{s}(x)=\hat{\mathbf{N}}(x) \hat{\mathbf{v}},
$$


with:

${ }^{272} \quad \hat{\mathbf{N}}(x)=\left[\begin{array}{cccccccccccc}N_{2}(x) & 0 & 0 & 0 & 0 & 0 & 0 & 0 & 0 & 0 & 0 & 0 \\ 0 & N_{4}(x) & N_{5}(x) & 0 & 0 & 0 & 0 & N_{4}(x) & 0 & 0 & N_{5}(x) & 0 \\ 0 & 0 & 0 & N_{3}(x) & 0 & 0 & N_{4}(x) & 0 & 0 & N_{5}(x) & 0 & 0 \\ 0 & 0 & 0 & 0 & -N_{4}(x) & -N_{5}(x) & 0 & 0 & N_{4}(x) & 0 & 0 & N_{5}(x) \\ 0 & 0 & 0 & 0 & 0 & 0 & 0 & N_{1}(x) & 0 & 0 & N_{2}(x) & 0 \\ 0 & 0 & 0 & 0 & 0 & 0 & 0 & 0 & N_{1}(x) & 0 & 0 & N_{2}(x)\end{array}\right]$.

273 where:

$$
\begin{array}{ll}
274 & N_{1}(x)=1-x / L, \\
275 & N_{2}(x)=x / L, \\
276 & N_{3}(x)=3 x^{2} / L^{2}-2 x^{3} / L^{3}, \\
277 & N_{4}(x)=x-2 x^{2} / L+x^{3} / L^{2}, \\
278 & N_{5}(x)=-x^{2} / L+x^{3} / L^{2} .
\end{array}
$$

To be noted is that the interpolation of the transverse displacements, bending rotations and shear strains, whose definition makes use of the additional shear strain DOFs, $\gamma_{y i / j}$ and $\gamma_{z i / j}$, coincides with that proposed in [35] for a 2D Timoshenko beam to avoid shear locking pathological problems [37]. Here, the interpolation is extended to the 3D formulation and referred to the basic element reference configuration. Moreover, fields $\gamma_{y}(x)$ and $\gamma_{z}(x)$ are used to describe cross-section warping, together with field $\chi_{x}(x)$. The latter results from the derivative with respect to $x$ of the torsional rotation $\theta_{x}(x)$, which is defined by exploiting the additional torsional strain DOFs, $\chi_{x i / j}$, [49].

By introducing Eq. (46) into Eq. (26), the generalized cross-section strains is related to the nodal basic displacement vector, and results as:

$$
\hat{\mathbf{e}}(x)=\hat{\mathbf{D}}(x) \hat{\mathbf{N}}(x) \hat{\mathbf{v}}=\hat{\mathbf{a}}(x) \hat{\mathbf{v}},
$$

where $\hat{\mathbf{a}}(x)$ is the cross-section strain compatibility matrix, resulting from the application of the differential operator $\hat{\mathbf{D}}(x)$ to the shape function matrix $\hat{\mathbf{N}}(x)$.

\subsubsection{Variational formulation and element governing equations}

The element governing equations are derived by invoking the stationarity of the total potential energy, which is written as:

$$
\hat{\Pi}\left(\hat{\mathbf{u}}_{s}(x)\right)=\int_{V} \varepsilon_{m}^{T}(x, y, z) \tilde{\mathbf{\sigma}}_{m}(x, y, z) d V-\hat{\mathbf{v}}^{T} \hat{\mathbf{q}}-\int_{L} \hat{\mathbf{u}}_{s}^{T}(x) \hat{\mathbf{q}}_{s}(x) d x,
$$

where $V$ is the element volume and $\hat{\mathbf{q}}_{s}(x)$ contains the loads distributed along the element axis. Parentheses (round brackets) indicate variable dependency. Vector $\hat{\mathbf{q}}_{s}(x)$ consists of four non-zero components: the axial load $q_{u}(x)$ 
parallel to $x$, the transverse loads $q_{v}(x)$ and $q_{w}(x)$ parallel to directions $y$ and $z$, respectively, and the torque $t_{x}(x)$ :

$$
\hat{\mathbf{q}}_{s}(x)=\left\{\begin{array}{llllll}
q_{u}(x) & q_{v}(x) & t_{x}(x) & q_{w}(x) & 0 & 0
\end{array}\right\}^{T} .
$$

By introducing Eqs. (28), (46) and (53) into Eq. (54), the total potential energy is expressed in terms of $\hat{\mathbf{v}}$ as follows:

$$
\hat{\Pi}(\hat{\mathbf{v}})=\hat{\mathbf{v}}^{T} \int_{L} \hat{\mathbf{a}}^{T}(x)\left[\int_{A(x)} \hat{\alpha}^{T}(y, z) \tilde{\boldsymbol{\sigma}}_{m}(x, y, z) d A\right] d x-\hat{\mathbf{v}}^{T} \hat{\mathbf{q}}-\hat{\mathbf{v}}^{T} \int_{L} \hat{\mathbf{N}}^{T}(x) \hat{\mathbf{q}}_{s}(x) d x,
$$

Moreover, by considering Eq. (30) and imposing the stationarity of $\hat{\Pi}$ with respect to $\hat{\mathbf{v}}$, the element equilibrium equation is derived, which reads:

$$
\hat{\mathbf{q}}=\int_{L} \hat{\mathbf{a}}^{T}(x) \hat{\mathbf{s}}(x) d x-\int_{L} \hat{\mathbf{N}}^{T}(x) \hat{\mathbf{q}}_{s}(x) d x,
$$

where second term on the right-hand side defines the element nodal forces due to the element loads, $\hat{\mathbf{q}}_{q}=\int_{L} \hat{\mathbf{N}}^{T}(x) \hat{\mathbf{q}}_{s}(x) d x$.

By applying the virtual work principle, considering the virtual nodal basic displacements $\hat{\mathbf{v}}=\hat{\mathbf{a}}_{v} \hat{\mathbf{u}}$, the definition of the element force vector $\hat{\mathbf{p}}$ is obtained in the form:

$$
\hat{\mathbf{p}}=\hat{\mathbf{a}}_{v}^{T} \hat{\mathbf{q}}+\hat{\mathbf{p}}_{r q},
$$

This collects the standard forces and couples, $\mathbf{p}_{i / j}$ and $\mathbf{m}_{i / j}$, work-conjugate to $\mathbf{u}_{i / j}$ and $\boldsymbol{\theta}_{i / j}$, and the additional generalized forces, $\beta$, work-conjugate to the warping DOFs, $\eta$, that is:

$$
\hat{\mathbf{p}}=\left\{\begin{array}{ll}
\mathbf{p}^{T} & \beta^{T}
\end{array}\right\}^{T},
$$

with:

$$
\mathbf{p}=\left\{\begin{array}{llll}
\mathbf{p}_{i}^{T} & \mathbf{m}_{i}^{T} & \mathbf{p}_{j}^{T} & \mathbf{m}_{j}^{T}
\end{array}\right\}^{T} .
$$

Vector $\hat{\mathbf{p}}_{r q}=\left\{\begin{array}{lllllll}\mathbf{p}_{r q}^{T} & 0 & 0 & 0 & 0 & 0 & 0\end{array}\right\}^{T}$ is the extended version of vector $\mathbf{p}_{r q}$, containing the basic reaction forces due to the distributed loads, $\hat{\mathbf{q}}_{s}(x)$. For uniform loads, this results as:

$$
\mathbf{p}_{r q}=-\left\{\begin{array}{lllllllllllll}
q_{u} L & q_{v} \frac{L}{2} & q_{w} \frac{L}{2} & t_{x} L & 0 & 0 & 0 & q_{v} \frac{L}{2} & q_{w} \frac{L}{2} & 0 & 0 & 0
\end{array}\right\}^{T} .
$$

The incremental form of Eq. (58) is written as:

$$
\Delta \hat{\mathbf{p}}=\hat{\mathbf{a}}_{v}^{T} \Delta \hat{\mathbf{q}}=\hat{\mathbf{a}}_{v}^{T} \hat{\mathbf{k}}_{v} \Delta \hat{\mathbf{v}}=\hat{\mathbf{a}}_{v}^{T} \hat{\mathbf{k}}_{v} \hat{\mathbf{a}}_{v} \Delta \hat{\mathbf{u}}=\hat{\mathbf{k}} \Delta \hat{\mathbf{u}},
$$

where $\hat{\mathbf{k}}=\hat{\mathbf{a}}_{v}^{T} \hat{\mathbf{k}}_{v} \hat{\mathbf{a}}_{v}$ is the element tangent stiffness matrix, with:

$$
\hat{\mathbf{k}}_{v}=\frac{\partial \hat{\mathbf{q}}}{\partial \hat{\mathbf{v}}}=\int_{L} \hat{\mathbf{a}}^{T}(x) \frac{\partial \hat{\mathbf{s}}(x)}{\partial \hat{\mathbf{e}}} \frac{\partial \hat{\mathbf{e}}(x)}{\partial \hat{\mathbf{v}}} d x=\int_{L} \hat{\mathbf{a}}^{T}(x) \hat{\mathbf{k}}_{s}(x) \hat{\mathbf{a}}(x) d x .
$$

This governs the incremental form of the element constitutive relationship expressed in the reference basic system and used in Eq. (62), that is:

$$
\Delta \hat{\mathbf{q}}=\hat{\mathbf{k}}_{v} \Delta \hat{\mathbf{v}}
$$




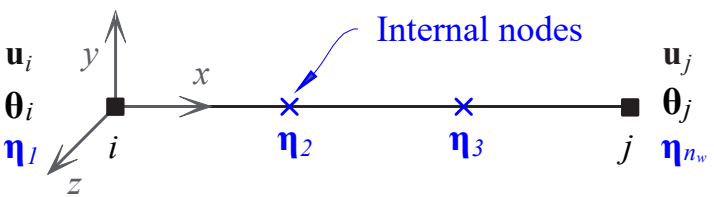
by Eq. (16), where now vector $\eta$ lists the warping DOFs of all the $n_{w}$ warping nodes, that is:

$$
\eta=\left\{\begin{array}{llll}
\eta_{1}^{T} & \eta_{2}^{T} & \ldots & \eta_{n_{w}}^{T}
\end{array}\right\}^{T}
$$

Figure 6: Standard (black) and warping (blue) nodal DOFs for the SWME model

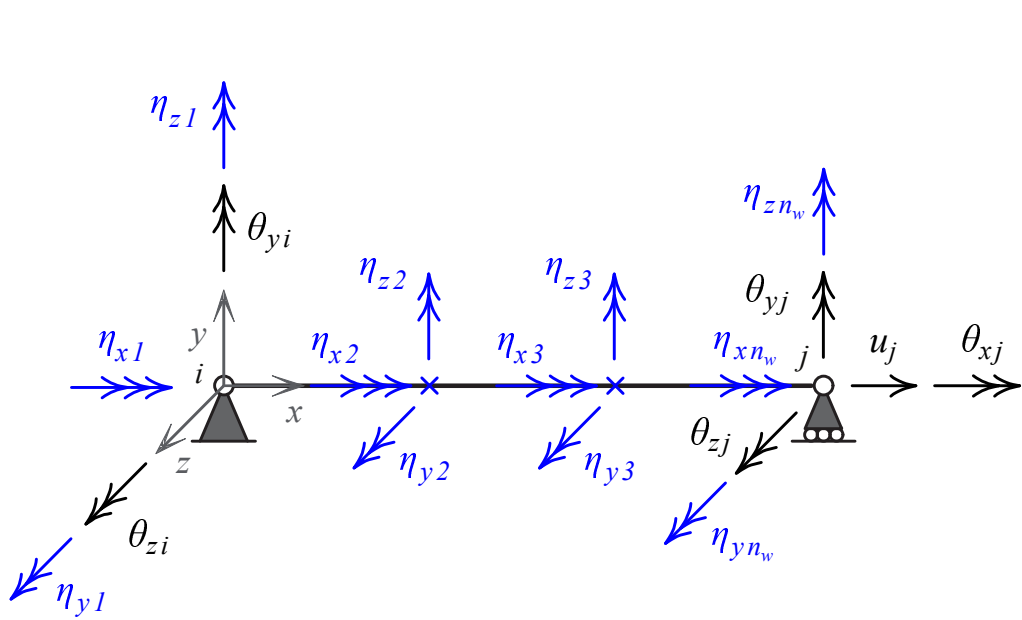

Figure 7: SWME reference configuration in the local system $(i ; x, y, z)$ with basic displacement (black) and warping (blue) DOFs

The SWME formulation is defined by introducing additional internal nodes, located along the element axis (Fig. 6), where supplementary warping DOFs are defined. In this work, without loss of generality, internal nodes are assumed as equally spaced along $x$, but any distribution can be considered. Hence, at the general node $n$, three DOFs are added, indicated as $\eta_{x n}, \eta_{y n}$ and $\eta_{z n}$ and collected in vector $\eta_{n}$. DOFs $\eta_{x n}$ are used to describe cross-section warping due to torsion, while $\eta_{y n}$ and $\eta_{z n}$ are used to describe warping due to shear. The nodal displacement vector is again expressed

With reference to the element basic configuration, the basic deformation displacement and warping DOFs are collected in the basic displacement vector $\hat{\mathbf{v}}$, resulting as in Eq. (19) (Fig. 7), with $\mathbf{I}$ and $\mathbf{0}$ having proper dimensions.

The internal force vector $\hat{\mathbf{q}}$, work-conjugate to $\hat{\mathbf{v}}$, results as in Eq. (20). However, in this case, vector $\beta$ lists the warping internal forces, $\boldsymbol{\beta}_{w n}$, defined at all the warping element nodes, that is:

$$
\beta=\left\{\begin{array}{llll}
\boldsymbol{\beta}_{w 1}^{T} & \beta_{w 2}^{T} & \ldots & \beta_{w n_{w}}^{T}
\end{array}\right\}^{T}
$$

The element constitutive law relating $\hat{\mathbf{q}}$ and $\hat{\mathbf{v}}$ is derived in Section 3.2.3. 


\subsubsection{Warping description and cross-section behavior}

Similar to the EVDE model, the warping displacement field $u_{w}(x, y, z)$ is assumed as defined in Eq. (22). However, differently from the Vlasov's assumption in Eq. (23), here $\eta_{s}(x)=\left\{\begin{array}{lll}\eta_{x}(x) & \eta_{y}(x) & \eta_{z}(x)\end{array}\right\}^{T}$ are considered as independent generalized cross-section fields, as proposed in Benscoter's theory [10, 21].

According to Eq. (6), the material strains $\boldsymbol{\varepsilon}_{m}(x, y, z)$ are determined as:

$$
\varepsilon_{m}(x, y, z)=\varepsilon_{r}(x, y, z)+\varepsilon_{w}^{\zeta}(x, y, z)+\varepsilon_{w}^{\eta}(x, y, z)=\alpha(y, z) \mathbf{e}(x)+\alpha_{\zeta}(y, z) \zeta_{s}(x)+\alpha_{\eta}(y, z) \eta_{s}(x)
$$

where two contributions are distinguished for the warping material strains, $\boldsymbol{\varepsilon}_{w}(x, y, z)$, that is $\boldsymbol{\varepsilon}_{w}^{\zeta}(x, y, z)=\boldsymbol{\alpha}_{\zeta}(y, z) \zeta_{s}(x)$ and $\boldsymbol{\varepsilon}_{w}^{\eta}(x, y, z)=\boldsymbol{\alpha}_{\eta}(y, z) \eta_{s}(x)$. Vector $\zeta_{s}(x)$ contains the derivatives of the warping displacements with respect to $x$, $\zeta_{s}(x)=\frac{\partial \eta_{s}(x)}{\partial x}$, i.e. the generalized strain quantities, $\zeta_{x}(x), \zeta_{y}(x)$ and $\zeta_{z}(x)$, introduced for the EVDE in Eq. (26), are here defined as the derivatives of the independent warping fields in $\eta_{s}(x)$. The matrices $\alpha_{\zeta}(y, z)$ and $\alpha_{\eta}(y, z)$ are two warping compatibility operators with size $3 \times 3$ and defined as:

$$
\alpha_{\zeta}(y, z)=\left[\begin{array}{c}
\mathbf{M}_{\eta}(y, z) \\
\mathbf{0} \\
\mathbf{0}
\end{array}\right], \quad \alpha_{\eta}(y, z)=\left[\begin{array}{c}
\mathbf{0} \\
\frac{\partial \mathbf{M}_{\eta}(y, z)}{\partial y} \\
\frac{\partial \mathbf{M}_{\eta}(y, z)}{\partial z}
\end{array}\right] .
$$

In addition to the standard beam internal forces $\mathbf{s}(x)$ (Eq. (8)), the virtual work equivalence, enforced alternatively with the virtual warping strains $\delta \varepsilon_{w}^{\zeta}(x, y, z)=\alpha_{\zeta}(y, z) \delta \zeta_{s}(x)$ and $\delta \varepsilon_{w}^{\eta}(x, y, z)=\alpha_{\eta}(y, z) \delta \eta_{s}(x)$, leads to the definition of beam internal forces, $\mathbf{b}_{w}(x)$ and $\mathbf{t}_{w}(x)$, associated to warping and resulting as:

$$
\mathbf{b}_{w}(x)=\int_{A(x)} \alpha_{\zeta}(y, z)^{T} \boldsymbol{\sigma}_{m}(x, y, z) d A, \quad \mathbf{t}_{w}(x)=\int_{A(x)} \alpha_{\eta}(y, z)^{T} \boldsymbol{\sigma}_{m}(x, y, z) d A .
$$

These are work-conjugate to $\zeta_{s}(x)$ and $\eta_{s}(x)$, respectively, and correspond to the quantities introduced for the EVDE model and called bi-moments and bi-shears.

Warping parameters $\eta_{s}(x)$ are interpolated along the element axis by exploiting warping DOFs collected in $\eta$ and by means of shape functions $N_{n}(x)$, that is $\eta_{s}(x)=\sum_{n=1}^{n_{w}} N_{n}(x) \eta_{n}$. Thus, Eq. (22) is now written as:

$$
u_{w}(x, y, z)=\sum_{n=1}^{n_{w}} N_{n}(x) \mathbf{M}_{\eta}(y, z) \eta_{n} .
$$

In the simplest version of the model, the additional warping nodes coincide with end nodes $i$ and $j\left(n_{w}=2\right)$ and functions $N_{n}(x)$ result as 2-node linear Lagrange polynomials. By contrast, when internal nodes are considered $\left(n_{w}>\right.$ 2), functions $N_{n}(x)$ result as $n_{w}$-node higher order Lagrange polynomials. By introducing Eq. (70) into Eq. (67), it results:

$$
\boldsymbol{\varepsilon}_{m}(x, y, z)=\alpha(y, z) \mathbf{e}(x)+\sum_{n=1}^{n_{w}}\left[\frac{\partial N_{n}(x)}{\partial x} \boldsymbol{\alpha}_{\zeta}(y, z)+N_{n}(x) \boldsymbol{\alpha}_{\eta}(y, z)\right] \eta_{n}
$$


By differentiating $\mathbf{s}(x), \mathbf{b}_{w}(x)$ and $\mathbf{t}_{w}(x)$ with respect to $\mathbf{e}(x), \zeta_{s}(x)$ and $\eta_{s}(x)$ and considering Eqs. (8), (69), (67) and (11), the cross-section generalized tangent stiffness matrices are obtained, resulting as:

$$
\begin{aligned}
\mathbf{k}_{s}(x) & =\frac{\partial \mathbf{s}(x)}{\partial \mathbf{e}}=\int_{A(x)} \boldsymbol{\alpha}^{T}(y, z) \mathbf{k}_{m}(x, y, z) \boldsymbol{\alpha}(y, z) d A, \\
\mathbf{k}_{s w}^{\zeta}(x) & =\frac{\partial \mathbf{s}(x)}{\partial \zeta_{s}}=\int_{A(x)} \boldsymbol{\alpha}^{T}(y, z) \mathbf{k}_{m}(x, y, z) \boldsymbol{\alpha}_{\zeta}(y, z) d A, \\
\mathbf{k}_{s w}^{\eta}(x) & =\frac{\partial \mathbf{s}(x)}{\partial \eta_{s}}=\int_{A(x)} \boldsymbol{\alpha}^{T}(y, z) \mathbf{k}_{m}(x, y, z) \boldsymbol{\alpha}_{\eta}(y, z) d A, \\
\mathbf{k}_{w s}^{\zeta}(x) & =\frac{\partial \mathbf{b}_{w}(x)}{\partial \mathbf{e}}=\int_{A(x)} \boldsymbol{\alpha}_{\zeta}^{T}(y, z) \mathbf{k}_{m}(x, y, z) \boldsymbol{\alpha}(y, z) d A, \\
\mathbf{k}_{w w}^{\zeta}(x) & =\frac{\partial \mathbf{b}_{w}(x)}{\partial \zeta_{s}}=\int_{A(x)} \boldsymbol{\alpha}_{\zeta}^{T}(y, z) \mathbf{k}_{m}(x, y, z) \boldsymbol{\alpha}_{\zeta}(y, z) d A, \\
\mathbf{k}_{w w}^{\zeta \eta}(x) & =\frac{\partial \mathbf{b}_{w}(x)}{\partial \eta_{s}}=\int_{A(x)} \boldsymbol{\alpha}_{\zeta}^{T}(y, z) \mathbf{k}_{m}(x, y, z) \boldsymbol{\alpha}_{\eta}(y, z) d A, \\
\mathbf{k}_{w s}^{\eta}(x) & =\frac{\partial \mathbf{t}_{w}(x)}{\partial \mathbf{e}}=\int_{A(x)} \boldsymbol{\alpha}_{\eta}^{T}(y, z) \mathbf{k}_{m}(x, y, z) \boldsymbol{\alpha}(y, z) d A, \\
\mathbf{k}_{w w}^{\eta \zeta}(x) & =\frac{\partial \mathbf{t}_{w}(x)}{\partial \zeta_{s}}=\int_{A(x)} \boldsymbol{\alpha}_{\eta}^{T}(y, z) \mathbf{k}_{m}(x, y, z) \boldsymbol{\alpha}_{\zeta}(y, z) d A, \\
\mathbf{k}_{w w}^{\eta}(x) & =\frac{\partial \mathbf{t}_{w}(x)}{\partial \eta_{s}}=\int_{A(x)} \boldsymbol{\alpha}_{\eta}^{T}(y, z) \mathbf{k}_{m}(x, y, z) \boldsymbol{\alpha}_{\eta}(y, z) d A .
\end{aligned}
$$

These govern the generalized cross-section constitutive relationships in incremental form that are derived by considering the incremental form Eq. (70), resulting as:

67

$$
\begin{aligned}
\Delta \mathbf{s}(x) & =\mathbf{k}_{s}(x) \Delta \mathbf{e}(x)+\sum_{n=1}^{n_{w}}\left[\frac{\partial N_{n}(x)}{\partial x} \mathbf{k}_{s w}^{\zeta}(x)+N_{n}(x) \mathbf{k}_{s w}^{\eta}(x)\right] \Delta \eta_{n}, \\
\Delta \mathbf{b}_{w}(x) & =\mathbf{k}_{w s}^{\zeta}(x) \Delta \mathbf{e}(x)+\sum_{n=1}^{n_{w}}\left[\frac{\partial N_{n}(x)}{\partial x} \mathbf{k}_{w w}^{\zeta}(x)+N_{n}(x) \mathbf{k}_{w w}^{\zeta \eta}(x)\right] \Delta \boldsymbol{\eta}_{n}, \\
\Delta \mathbf{t}_{w}(x) & =\mathbf{k}_{w s}^{\eta}(x) \Delta \mathbf{e}(x)+\sum_{n=1}^{n_{w}}\left[\frac{\partial N_{n}(x)}{\partial x} \mathbf{k}_{w w}^{\eta \zeta}(x)+N_{n}(x) \mathbf{k}_{w w}^{\eta}(x)\right] \Delta \boldsymbol{\eta}_{n} .
\end{aligned}
$$

\subsubsection{Cross-section stress interpolation}

As opposed to the EVDE formulation, where only standard and warping displacements are interpolated along the element axis, according to the mixed formulation [40, 41, 50], the SWME adopts independent interpolations for the generalized cross-section stresses along $x$, together with fields $\eta_{s}(x)$ (Eq. (70)). Hence, in line with the equilibrated beam formulation, the generalized cross-section stress vector $\mathbf{s}(x)$, i.e. the beam internal force vector, is related to the element basic forces $\mathbf{q}$ by the equilibrium conditions enforced in strong form, and results as:

$$
\mathbf{s}(x)=\mathbf{b}(x) \mathbf{q}+\mathbf{s}_{q}(x),
$$


where $\mathbf{b}(x)$ is the equilibrium matrix [45], defined as:

$$
\mathbf{b}(x)=\left[\begin{array}{cccccc}
1 & 0 & 0 & 0 & 0 & 0 \\
0 & \frac{x}{L}-1 & \frac{x}{L} & 0 & 0 & 0 \\
0 & -\frac{1}{L} & -\frac{1}{L} & 0 & 0 & 0 \\
0 & 0 & 0 & 1 & 0 & 0 \\
0 & 0 & 0 & 0 & \frac{x}{L}-1 & \frac{x}{L} \\
0 & 0 & 0 & 0 & \frac{1}{L} & \frac{1}{L}
\end{array}\right],
$$

and $\mathbf{s}_{q}(x)$ contains the generalized section stresses due to distributed loads $\mathbf{q}_{s}(x)$, arranged as:

$$
\mathbf{q}_{s}(x)=\left\{\begin{array}{llllll}
q_{u}(x) & 0 & q_{v}(x) & t_{x}(x) & 0 & q_{w}(x)
\end{array}\right\}^{T} .
$$

In case of uniformly distributed loadings, $\mathbf{s}_{q}(x)$ results as:

$$
\mathbf{s}_{q}(x)=\left\{\begin{array}{lllll}
{[L-x] q_{u}} & \frac{x}{2}[x-L] q_{v} & {\left[\frac{L}{2}-x\right] q_{v}} & {[L-x] t_{x}} & \frac{x}{2}[L-x] q_{w}
\end{array}\left[\frac{L}{2}-x\right] q_{w}\right\}^{T} .
$$

Eq. (84) is obtained as solution of the standard differential equilibrium equations of a straight Timoshenko beam [51] written in compact form as:

$$
\mathbf{D}^{*}(x) \mathbf{s}(x)+\mathbf{q}_{s}(x)=\mathbf{0},
$$

being $\mathbf{D}^{*}(x)$ the equilibrium operator, self-adjoint to $\mathbf{D}(x)$ (Eq. (7)). Generalization to curved beams were proposed resulting in very efficient formulations [43] with respect to the classical displacement-based approach.

\subsubsection{Variational formulation and element governing equations}

The element governing equations are derived by invoking the stationarity of a modified four-field $\mathrm{Hu}-\mathrm{Washizu}$ variational functional [52, 53]. This is defined by assuming $\mathbf{u}_{r}(x, y, z), \boldsymbol{\varepsilon}_{m}(x, y, z), \boldsymbol{\sigma}_{m}(x, y, z)$ and $u_{w}(x, y, z)$ as independent fields and is written as:

$$
\begin{aligned}
\Pi\left(\mathbf{u}_{r}, \boldsymbol{\varepsilon}_{m}, \boldsymbol{\sigma}_{m}, u_{w}\right) & =\int_{V} \boldsymbol{\varepsilon}_{m}^{T}(x, y, z) \tilde{\boldsymbol{\sigma}}_{m}(x, y, z) d V+\int_{V} \boldsymbol{\sigma}_{m}^{T}(x, y, z)\left\{\boldsymbol{\varepsilon}_{m}\left(\mathbf{u}_{m}(x, y, z)\right)-\boldsymbol{\varepsilon}_{m}(x, y, z)\right\} d V \\
& -\mathbf{u}^{T} \mathbf{p}-\eta^{T} \beta-\int_{L} \mathbf{u}_{s}^{T}(x) \mathbf{q}_{s}(x) d x .
\end{aligned}
$$

Parentheses (round brackets) indicate variable dependency.

The introduction of $\mathbf{u}_{r}(x, y, z)=\alpha(y, z) \mathbf{u}_{s}(x)$ and Eqs. (8), (70) and (71) into Eq. (89), permits to express the functional in terms of fields $\mathbf{u}_{s}(x), \mathbf{e}(x), \mathbf{s}(x)$ and $\eta$, that is:

$$
\begin{aligned}
\Pi\left(\mathbf{u}_{s}, \mathbf{e}, \mathbf{s}, \boldsymbol{\eta}\right) & =\int_{V} \boldsymbol{\varepsilon}_{m}^{T}\left(\mathbf{e}(x), u_{w}(\boldsymbol{\eta}), x, y, z\right) \tilde{\boldsymbol{\sigma}}_{m}\left(\boldsymbol{\varepsilon}_{m}(x, y, z)\right) d V+\int_{L} \mathbf{s}^{T}(x)\left[\mathbf{e}\left(\mathbf{u}_{s}(x)\right)-\mathbf{e}(x)\right] d x \\
& -\mathbf{u}^{T} \mathbf{p}-\boldsymbol{\eta}^{T} \beta-\int_{L} \mathbf{u}_{s}^{T}(x) \mathbf{q}_{s}(x) d x,
\end{aligned}
$$

By enforcing the stationarity of $\Pi$ with respect to the four independent fields $\mathbf{u}_{s}(x), \mathbf{e}(x), \mathbf{s}(x), \eta$, the element governing equations are derived as follows: 
- Considering Eqs. (8) and (67), it results as [52, 45]:

$$
\begin{aligned}
\frac{\partial \Pi\left(\mathbf{u}_{s}, \mathbf{e}, \mathbf{s}, \boldsymbol{\eta}\right)}{\partial \mathbf{e}} & =\frac{\partial}{\partial \mathbf{e}}\left\{\int_{V} \mathbf{e}^{T}(x) \boldsymbol{\alpha}^{T}(y, z) \tilde{\boldsymbol{\sigma}}_{m}\left(\boldsymbol{\varepsilon}_{m}(x, y, z)\right) d V-\int_{L} \mathbf{e}^{T}(x)\left[\int_{A(x)} \boldsymbol{\alpha}^{T}(y, z) \boldsymbol{\sigma}_{m}(x, y, z) d A\right] d x\right\} \\
& =\int_{V} \boldsymbol{\alpha}^{T}(y, z)\left[\tilde{\boldsymbol{\sigma}}_{m}\left(\boldsymbol{\varepsilon}_{m}(x, y, z)\right)-\boldsymbol{\sigma}_{m}(x, y, z)\right] d V=\mathbf{0} .
\end{aligned}
$$

This is satisfied if the material constitutive law in Eq. (9) holds, as the term in braces vanish in this case.

- Considering the equilibrium condition expressed by Eq. (84), it results as:

$$
\frac{\partial \Pi\left(\mathbf{u}_{s}, \mathbf{e}, \mathbf{s}, \boldsymbol{\eta}\right)}{\partial \mathbf{s}}=\frac{\partial}{\partial \mathbf{q}}\left\{\mathbf{q}^{T} \int_{L} \mathbf{b}^{T}(x)\left[\mathbf{e}\left(\mathbf{u}_{s}(x)\right)-\mathbf{e}(x)\right] d x\right\}=\int_{L} \mathbf{b}^{T}(x) \mathbf{e}\left(\mathbf{u}_{s}(x)\right)-\int_{L} \mathbf{b}^{T}(x) \mathbf{e}(x) d x=\mathbf{0} .
$$

After introducing Eq. (7), the first integral is integrated by parts and only boundary quantities result as non-zero terms. These correspond to the element deformation displacements $\mathbf{v}$. Hence, the standard element compatibility condition, enforced in the weak form, is obtained, i.e:

$$
\mathbf{v}=\int_{L} \mathbf{b}^{T}(x) \mathbf{e}(x) d x .
$$

$$
\frac{\partial \Pi\left(\mathbf{u}_{s}, \mathbf{e}, \mathbf{s}, \boldsymbol{\eta}\right)}{\partial \mathbf{u}_{s}}=\frac{\partial}{\partial \mathbf{u}_{s}}\left\{\int_{L} \mathbf{e}^{T}\left(\mathbf{u}_{s}(x)\right) \mathbf{s}(x) d x-\mathbf{u}^{T} \mathbf{p}-\int_{L} \mathbf{u}_{s}^{T}(x) \mathbf{q}_{s}(x) d x\right\}=\mathbf{0} .
$$

As for Eq. (92), after introducing Eq. (7), the first integral is integrated by parts and, by introducing Eq. (88) to manipulate the resulting derivatives of internal forces $\mathbf{s}(x)$, the integrals are eliminated. The stationarity condition enforced for the remaining boundary terms is re-written as:

$$
\frac{\partial \Pi\left(\mathbf{u}_{s}, \mathbf{e}, \mathbf{s}, \eta\right)}{\partial \mathbf{u}_{s}}=\frac{\partial}{\partial \mathbf{u}_{s}}\left\{\left[\mathbf{u}_{s}^{T}(x) \mathbf{s}(x)\right]_{0}^{L}-\mathbf{u}^{T} \mathbf{p}\right\}=\frac{\partial}{\partial \mathbf{u}}\left\{\mathbf{u}^{T}\left[\mathbf{a}_{v}^{T} \mathbf{q}-\mathbf{p}_{r p}\right]-\mathbf{u}^{T} \mathbf{p}\right\}=\mathbf{0}
$$

and gives the element equilibrium conditions expressed in weak form, i.e.:

$$
\mathbf{p}=\mathbf{a}_{v}^{T} \mathbf{q}+\mathbf{p}_{r p} .
$$

$$
\frac{\partial \Pi\left(\mathbf{u}_{s}, \mathbf{e}, \mathbf{s}, \boldsymbol{\eta}\right)}{\partial \eta}=\int_{V} \frac{\boldsymbol{\varepsilon}_{m}^{T}\left(\mathbf{e}(x), u_{w}(\boldsymbol{\eta}), x, y, z\right)}{\partial \eta} \tilde{\boldsymbol{\sigma}}_{m}\left(\boldsymbol{\varepsilon}_{m}(x, y, z)\right) d V-\boldsymbol{\beta}=\mathbf{0} .
$$

Considering Eq. (9) and introducing Eqs. (69) and (71), the integral is expressed in terms of warping beam internal forces and the cross-section equilibrium conditions related to the warping are obtained, i.e.:

$$
\beta=\int_{L}\left[\begin{array}{c}
\frac{\partial N_{1}(x)}{\partial x} \\
\ldots \\
\frac{\partial N_{n_{w}}(x)}{\partial x}
\end{array}\right] \mathbf{b}_{w}(x) d x+\int_{L}\left[\begin{array}{c}
N_{1}(x) \\
\ldots \\
N_{n_{w}}(x)
\end{array}\right] \mathbf{t}_{w}(x) d x .
$$




\subsection{Enriched Warping Mixed Element (EWME) formulation}

The EWME formulation presented in $[43,45]$ can be seen as the generalized version of the SWME, enriched by introducing additional warping DOFs at the element warping nodes located both at the ends and along the element axis. In this case, an arbitrary number, $m_{w}$, of DOFs are considered at each node and collected in vector $\eta_{n}($ Fig. 6). These are located at uniformly distributed points over the cross-section (Fig. 8) and represent their out-of-plane warping displacement, namely $u_{w n m}$. Hence, the nodal displacement vector result as in Eq. (16), with $\eta$ arranged according to Eq. (65).

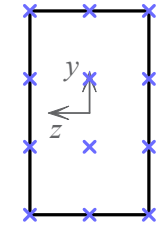

Rectangular

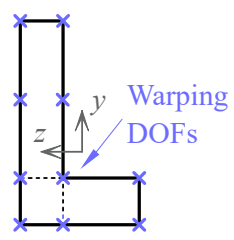

L-shaped

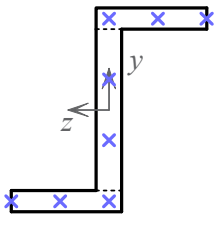

S-shaped

Figure 8: Examples of EWME warping DOFs for typical cross-sections

Warping DOFs are used to interpolate warping displacement field $u_{w}(x, y, z)$ in the element volume. As opposed to the SWME, where cross-section warping profiles $\mathbf{M}_{\eta}(y, z)$ are assigned and only the interpolation along the element axis is performed, in this case, the interpolation considers both the variation along $x$ and that over the cross-section. Hence, Eq. (4) is written as:

$$
u_{w}(x, y, z)=\sum_{n=1}^{n_{w}} N_{n}(x)\left[\sum_{m=1}^{m_{w}} M_{m}(y, z) u_{w n m}\right]=\sum_{n=1}^{n_{w}} N_{n}(x) \mathbf{M}(y, z) \eta_{n},
$$

where $\mathbf{M}(y, z)$ is a row vector containing 2D Lagrange polynomials $M_{m}(y, z)$ used to interpolate $u_{w}(x, y, z)$ over the cross-section plane. In a proposed modified version of the model [54], the adoption of Hermite polynomials is also explored for shape functions $M_{m}(y, z)$, defined by introducing the derivatives of $u_{w}(x, y, z)$ along $y$ and $z$ as supplementary warping DOFs. Although this approach significantly reduces the number of DOFs involved in the interpolation, it is not applicable to very thin-walled geometries [53] and, thus, only the case of Lagrange polynomials is considered in this work.

The material strains, $\boldsymbol{\varepsilon}_{m}(x, y, z)$, are derived according to Eq. (71) where matrices $\boldsymbol{\alpha}_{\zeta}(y, z)$ and $\boldsymbol{\alpha}_{\eta}(y, z)$ have, here, size $3 \times m_{w}$ and are defined as:

$$
\boldsymbol{\alpha}_{\zeta}(y, z)=\left[\begin{array}{c}
\mathbf{M}(y, z) \\
\mathbf{0} \\
\mathbf{0}
\end{array}\right], \quad \boldsymbol{\alpha}_{\eta}(y, z)=\left[\begin{array}{c}
\mathbf{0} \\
\frac{\partial \mathbf{M}(y, z)}{\partial y} \\
\frac{\partial \mathbf{M}(y, z)}{\partial z}
\end{array}\right] .
$$

Hence, by manipulating the cross-section and material quantities, as done for the SWME (Section 3.2.2), the generalized cross-section stresses, $\mathbf{b}_{w}(x)$ and $\mathbf{t}_{w}(x)$, associated to warping are derived as in Eq. (69). These play the same role as the bi-moments and bi-shears introduced for the SWME and EVDE. 
The other fundamental relationships and the element governing equations are obtained through similar derivations performed for the SWME and are not reported here for brevity. The element governing equations coincide with those standard for a force-based variational formulation, i.e. the element nodal equilibrium (Eq. (96)), the constitutive material law (Eq. (9)) and the element compatibility relation in weak form (Eq. (93)). In addition, the fourth equation enforcing the cross-section equilibrium for the warping forces is obtained, which reads as Eq. (98). However, in this case, $\beta$ (Eq. (66)) collects all the warping forces, $p_{w n m}$, work-conjugate to the warping DOFs, $u_{w n m}$. Complete details of the element formulation are described in [43, 45, 53].

\section{Numerical applications}

To test the performances of the proposed models, the mechanical response of thin-walled beams with open and closed, as well as symmetric and non-symmetric, cross-sections is numerically reproduced, under the assumption of linear elastic constitutive behavior and small displacements and strains. After presenting the method used to evaluate warping functions $M_{\eta x}(y, z), M_{\eta y}(y, z)$ and $M_{\eta z}(y, z)$ (Eq. (22)), numerical tests of three cantilever beams and a L frame are presented under pure torsional or coupled torsional and shear/bending actions. Results are compared with analytical solutions obtained with simplified and higher order models and the influence of the different warping descriptions adopted in the proposed beam formulations is discussed.

A fiber discretization is adopted to evaluate the cross-section response $[43,55]$, that is to solve all integrals defined over the cross-section plane. As linear elastic material response is assumed, cross-section fibers are defined according to the Gauss-Legendre 2D quadrature rule, so that exact solution of the integral is obtained [53]. Similarly, GaussLobatto 1D quadrature rule is used to solve the integrals defined along the element axis, so that two of the quadrature points are located at the element end nodes permitting to directly control the response of the end cross-sections.

\subsection{Numerical evaluation of the warping functions}

A numerical procedure is adopted to evaluate functions $M_{\eta x}(y, z), M_{\eta y}(y, z)$ and $M_{\eta z}(y, z)$ describing the warping displacement variation over the beam cross-section (Eq. (22)) for EVDE and SWME models. This relies on the linear elastic solution obtained by means of the EWME model for a unit length beam, adopting same cross-section geometry and constitutive behavior of the element to be analyzed. The beam is modeled adopting one FE, under the assumption of uniform warping along the element axis, i.e. by placing additional warping DOFs only at one element node, i.e. $n_{w}=1$, where warping displacements are assumed as free. Then, unit torsional curvature, $\chi_{x}$, and unit shear strains, $\gamma_{y}$ and $\gamma_{z}$, are alternatively imposed along the beam. To do so, unit torsional rotation, $\theta_{x j}$, and transverse displacements, $v_{j}$ and $w_{j}$, are alternatively imposed at node $j$, while assuming all other rigid cross-section displacements and rotations as prevented at both element ends (Fig. 9). The corresponding warping DOFs, $\eta_{1}$, are computed and, by applying Eq. (99), warping profiles of displacement $u_{w}(x, y, z)$ are determined over the beam cross-section and expressed by means of selected analytical shape functions. These warping profiles correspond to functions $M_{\eta x}(y, z), M_{\eta y}(y, z)$ 
and $M_{\eta z}(y, z)$, respectively. Their derivatives and integrals required in EVDE and SWME governing equations are computed by differentiation and integration of the 2D Lagrange polynomials $M_{m}(y, z)$ used in Eq. (99).

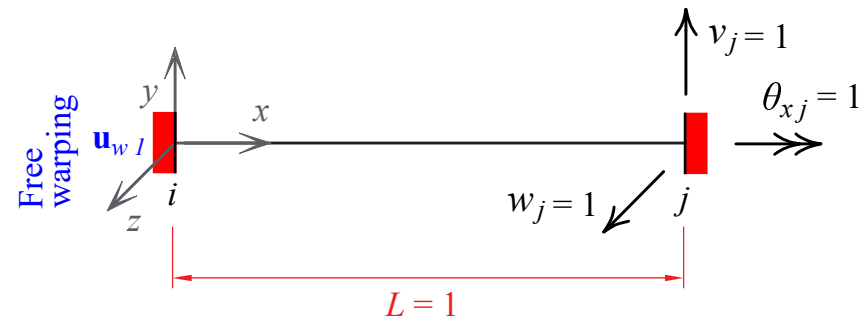

Figure 9: Numerical modeling scheme to evaluate warping functions $M_{\eta x}(y, z), M_{\eta y}(y, z)$ and $M_{\eta z}(y, z)$ $v=0.3$ are assumed for the material elastic constitutive law.

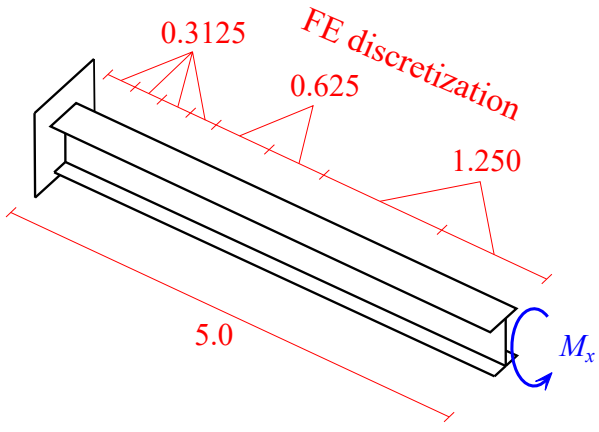

(a) numerical results $[43,53]$.

\subsection{Twist of a long I-shape cantilever}

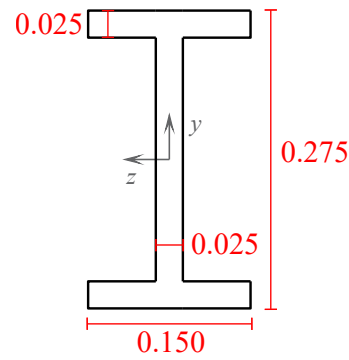

(b)

Figure 10: I-shaped cantilever: (a) static scheme with FE element mesh description and (b) cross-section geometry (all dimensions in meters)

Examples of warping functions determined for typical cross-section geometries are shown in Fig. 5. These are obtained by assuming the warping DOF scheme over the cross-section (Fig. 9) such that the interpolation of $u_{w}(x, y, z)$ results as cubic along both in-plane directions for the rectangular cross-section and cubic along webs and flanges for other geometries. For the latter, quadratic interpolation is assumed across the membrature thickness.

These interpolation schemes are adopted to determine the warping functions in all numerical tests performed in this work. However, lower or higher order interpolations can be, in general, used according to desired accuracy of the

The first test analyzes the response of the I-shaped cantilever depicted in Fig. 10, which was studied in the works by Tralli [12], Kim \& Kim [15] and Back \& Will [19]. Warping is assumed as fully restrained at the fixed end and a torsional couple, $M_{x}=25 \mathrm{kNm}$, is applied at the free end. Young's modulus $E=200000 \mathrm{MPa}$ and Poisson ratio

As usual for doubly-symmetric cross-sections, torsional and shear/bending behavior are uncoupled and, thus, null shear forces are expected along the beam. 
The specimen is first modeled by discretizing the beam with equal length FEs (uniform mesh), based on the proposed enhanced formulations. As the thickness of the membrature is significant compared to the cross-section size, for the EWME the warping interpolation scheme over the cross-section assumes quadratic variation across web and flange thickness, while cubic interpolation is assumed in the other directions. Hence, the interpolation scheme coincides with that adopted to compute the warping function $M_{\eta x}(y, z), M_{\eta y}(y, z)$ and $M_{\eta z}(y, z)$ to be introduced in the EVDE and SWME. The total number of warping DOFs used at each cross-section is $m_{w}=60$. For both the SWME and EWME, two schemes are considered for the warping interpolation along the element axis, i.e. $n_{w}=3$ and $n_{w}=4$, corresponding to quadratic and cubic variation, respectively. To be noted is that the EVDE considers cubic variation of the torsional rotation along the FE axis and, thus, according to Eqs. (22) and (23), quadratic variation of the warping displacement in this direction.

Fig. 11 shows the convergence of the solution in terms of torsional rotation at the free end, $\theta_{x}(L)$, as the number of FEs along the beam axis is increased, and compares the results with those reported in [12, 15, 19]. Green curve

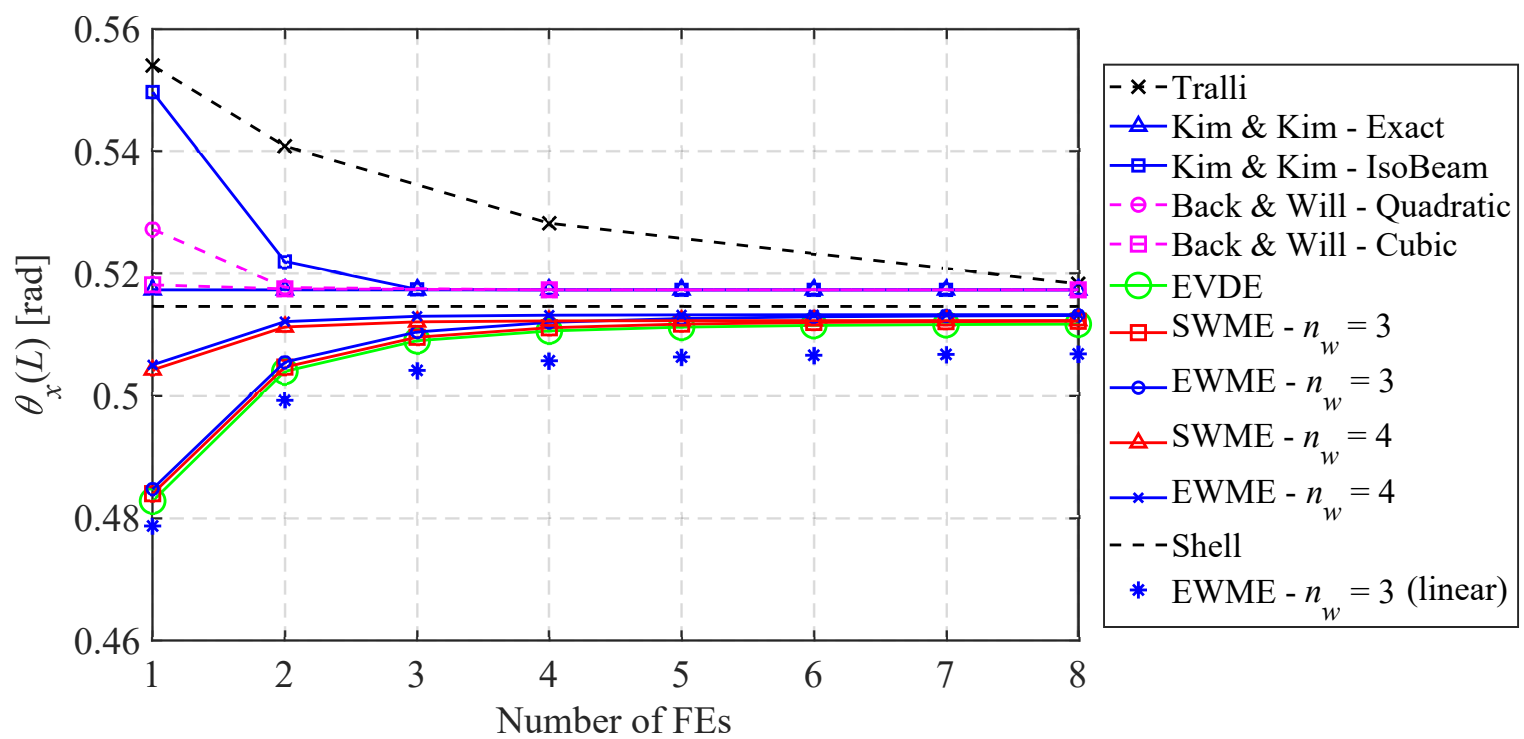

Figure 11: I-shaped cantilever: convergence of the solution for the uniform mesh in terms of torsional rotation at the free end, $\theta_{x}(L)$

with circles represents the solution obtained with EVDE, red curves with squares and triangles correspond to the solutions obtained with SWME, for $n_{w}=3$ and $n_{w}=4$, respectively, and blue curves with circles and crosses denote the solutions obtained with EWME, for $n_{w}=3$ and $n_{w}=4$, respectively. As expected, all these converge to similar results. Indeed, for doubly-symmetric open cross-sections, warping interpolation provided by Eqs. (22) and (23) suffices to describe the cross-section out-of-plane deformations and enhanced theories are not required. However, models assuming cubic warping variation along the element axis (SWME and EWME with $\left.n_{w}=4\right)$ exhibit higher performances than those considering quadratic interpolation of $u_{w}(x, y, z)$ (SWME and EWME with $n_{w}=3$ and EVDE). 
Similar trends are observed for models in $[12,15,19]$. Their solutions are in good agreement with those obtained with the proposed models. Black curve with crosses represents the solution reported in [12], while blue curves with triangles and squares correspond to the solutions reported in [15] and obtained with an exact analytical and a numerical approach, respectively. The latter relies on a 2-node isoparametric beam formulation, with linear warping variation along the element axis. Magenta curves with circles and asterisks represent the solutions reported in [19] and obtained with a beam formulation that assumes quadratic and cubic warping variation along the element axis, respectively.

For comparison, Fig. 11 also shows the result obtained with a shell FE model (dashed black horizontal line), which provides a solution almost coinciding with those obtained with the proposed models. The shell model adopts 4-nodes Mindlin shell FEs [56], properly formulated to avoid shear-locking problems. These have size equal to $0.00625 \mathrm{~m}$ along the cross-section mid-line and $0.025 \mathrm{~m}$ along the cantilever axis direction. Moreover, to satisfy the in-plane rigid cross-section assumption, also adopted for the beam models, the rigid diaphragm constraint is used in the crosssection plane. Couple $M_{x}$ is introduced by applying two opposite forces at the end cross-section, acting in the plane of the flanges, and the cross-section rotation is determined as the average value obtained by dividing the transverse displacements of the web nodes by their distance from the cross-section centroid [57].

To show the influence of the warping interpolation assumed over the beam cross-section, the solution obtained with the EWME for $n_{w}=3$ but assuming linear warping interpolation across the membrature thickness is plotted (blu stars). Cubic variation is still considered along web and flanges. As cross-section thickness is almost $1 / 3$ of cross-section width, warping variation across the thickness is important for this specimen and the linear interpolation assumption leads to stiffer solutions than those obtained with quadratic shape functions. To be noted is also that, if linear interpolation were used to evaluate warping functions $M_{\eta x}(y, z), M_{\eta y}(y, z)$ and $M_{\eta z}(y, z)$ for EVDE and SWME, these would give similar results as those obtained with this latter EWME model, that is the curves for the free end rotation would converge to the same value, as occurs for the plotted quadratic interpolation cases.

Following the studies reported in [12], similar analyses are conducted, yet adopting a non-uniform FE mesh discretization for the cantilever, as depicted in Fig. 10(a). This considers short, regular and long FEs, with length equal to $0.3125 \mathrm{~m}, 0.625 \mathrm{~m}$ and $1.250 \mathrm{~m}$, respectively, to better capture variation of the warping displacement near the fixed end and that of the axial stress due to shear-lag effect. Figs. 12(a) and 12(b) show the variation along the cantilever axis of the torsional rotation, $\theta_{x}$, and maximum axial stress, $\sigma_{x x}^{\max }$, obtained with the proposed models (solid curves with symbols) and compares these with the solution obtained in [12] adopting the same mesh (black crosses) and the shell model (dashed black curves). As indicated in Fig. 12(b), $\sigma_{x x}^{\max }$ occurs at the flange tips, where higher values of axial strain are induced due to the warping restraints placed at the fixed end, and vanishes moving toward the free end of the cantilever. These variation clearly describes the influence of warping restraints placed at the fixed end. These strongly affect the element response, by increasing the torsional stiffness of the cantilever. This behavior is perfectly described by the proposed beam formulations that highly agree with the shell model. 


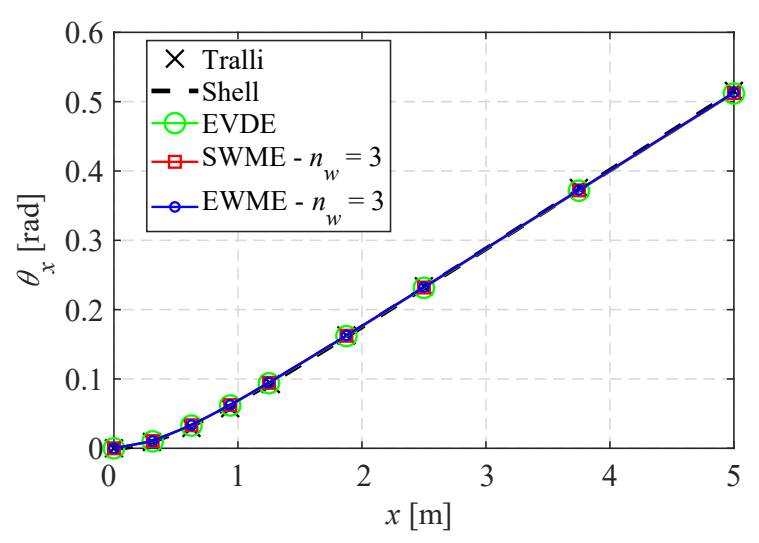

(a)

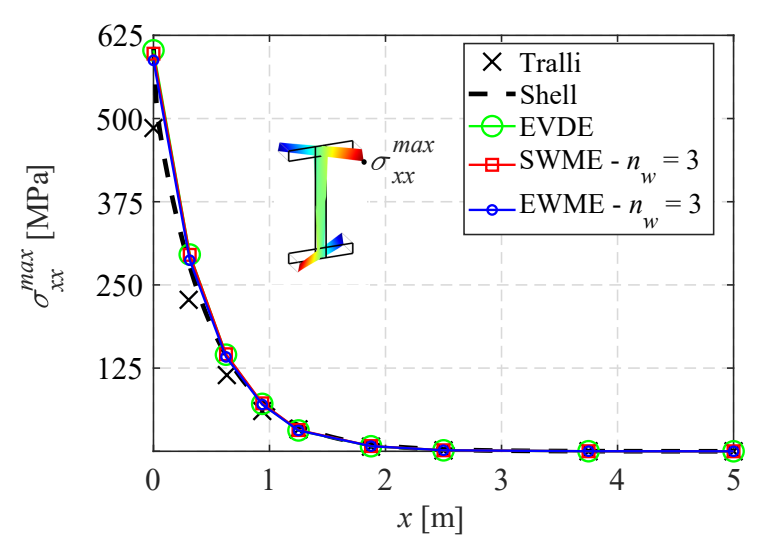

(b)

Figure 12: I-shaped cantilever (non-uniform mesh): axial variation of (a) torsional rotation, $\theta_{x}$, and (b) maximum axial stress, $\sigma_{x x}^{m a x}$, evaluated at the tip of the flanges due to shear-lag effect

\subsection{Twist of a short $C$-shape cantilever}

The second test analyzes the behavior of a cantilever beam with C-shaped cross-section subjected to a concentrated torsional couple at the free end, equal to $M_{x}=1000 \mathrm{kNm}$, and with warping restrained at the fixed end. Specimen geometry is depicted in Fig. 13 and material behavior is defined by assuming Young's modulus $E=30 \mathrm{MPa}$ and Poisson ratio $v=0.154$. In this test, torsional and bending/shear effects are coupled $[12,15,19,33]$ and secondary shear stresses are expected to play an important role in the beam response.

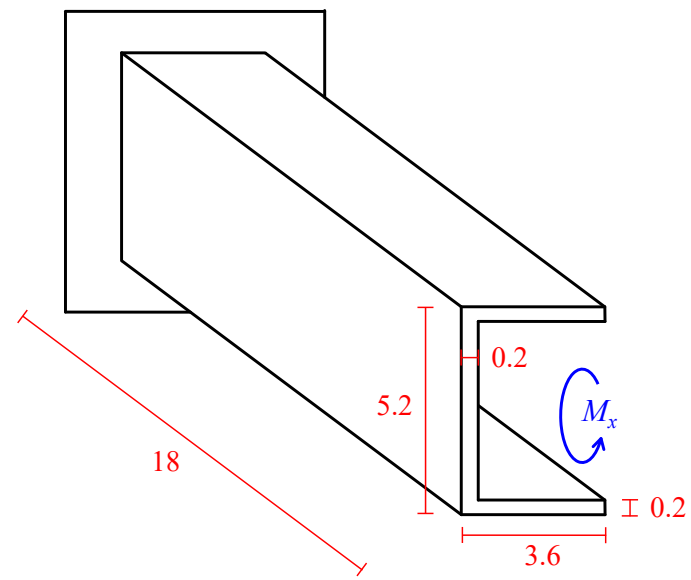

Figure 13: C-shaped cantilever: static scheme and specimen geometry (all dimensions in meters)

The beam is modeled by adopting a uniform mesh made of six FEs based on the proposed formulations. For the SWME and EWME models, parabolic warping interpolation is assumed along the element axis, i.e. $n_{w}=3$. To be noted is that same order of interpolation is considered by the EVDE model for torsional warping, but linear interpolation is considered for shear warping (see Eqs. (22), (23) and (46)). For the EWME, warping interpolation is 
assumed as cubic along web and flanges and parabolic across the membrature thickness for a total number of warping DOFs at each cross-section equal to $m_{w}=42$.

Fig. 14(a) shows the variations along the element axis of the torsional rotation, $\theta_{x}$, (green curve with circles for the EVDE, red curve with squares for the SWME and blue curve with dots for the EWME) and compares these with the analytical solution reported in [15] (black crosses) that includes the effects of the secondary shear stresses. A very small difference is observed between the proposed model results, that is the EVDE and SWME provide slightly stiffer solutions than the EMWE. However, this latter perfectly agrees with the reference analytical approach, as the EWME is based on a richer description of the warping displacements over the element cross-section than the other two formulations. Same behavior is shown in Fig. 14(b), where the convergence of the solution is plotted in terms of free end torsional rotation, $\theta_{x}(L)$, for increasing number of FEs. As expected, EVDE (green curve with circles), considering lower order interpolation for shear warping, exhibits worse performances than SWME (red curve with squares) and EWME (blue curve with dots). For this latter, one FE suffices to model beam. Moreover, as expected, due the coarser warping description, EVDE results converge to a value that is close to Vlasov's analytical solution, indicated by the black solid line, while higher free end rotation is provided by SWME and EWME at convergence.

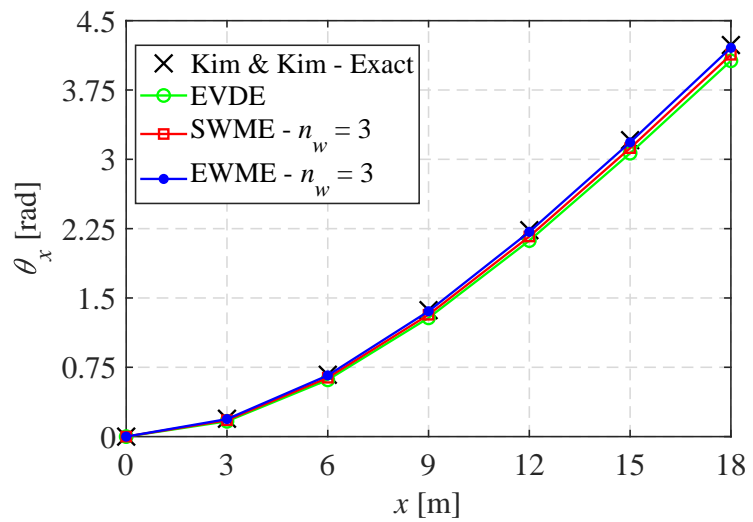

(a)

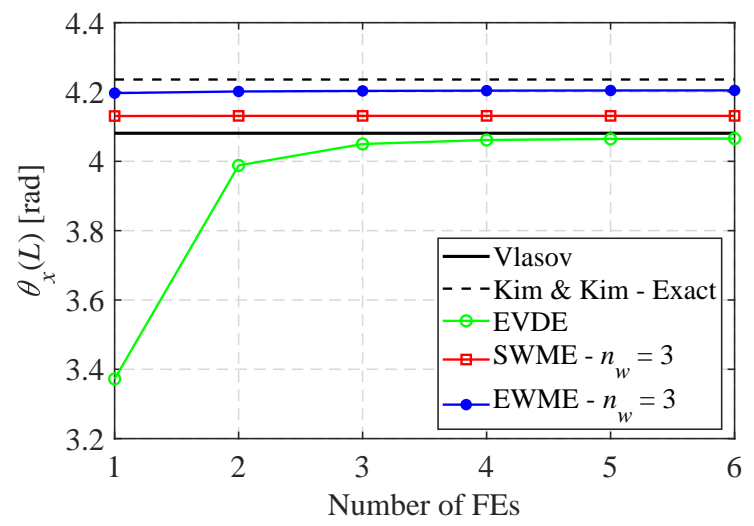

(b)

Figure 14: C-shaped cantilever: (a) axial variation of torsional rotation, $\theta_{x}$, and (b) convergence of the solution in terms of free end torsional rotation, $\theta_{x}(L)$, for the proposed models

Figs. 15(a) and 15(b) plot the distributions of the axial and tangential stresses, $\sigma_{x x}$ and $\tau_{x z}$, respectively, evaluated with the three proposed models at $4.5 \mathrm{~m}$ from the fixed end, being $z$ the direction parallel to the cross-sections web. As shown, all formulations well reproduce the distribution of the axial stress $\sigma_{x x}$ with no remarkable difference in values among the models. This is due only to shear-lag effect, that is to the warping restraints applied at the fixed end that produce relevant strains in the axial direction. Only EWME correctly describes the trend of the tangential stress $\tau_{x z}$, and, thus, the influence of secondary shear stresses, as can be observed by comparing the results with those reported in [33]. Due to warping variation across the membrature thickness, $\tau_{x z}$ attains maximum values at the external edges 


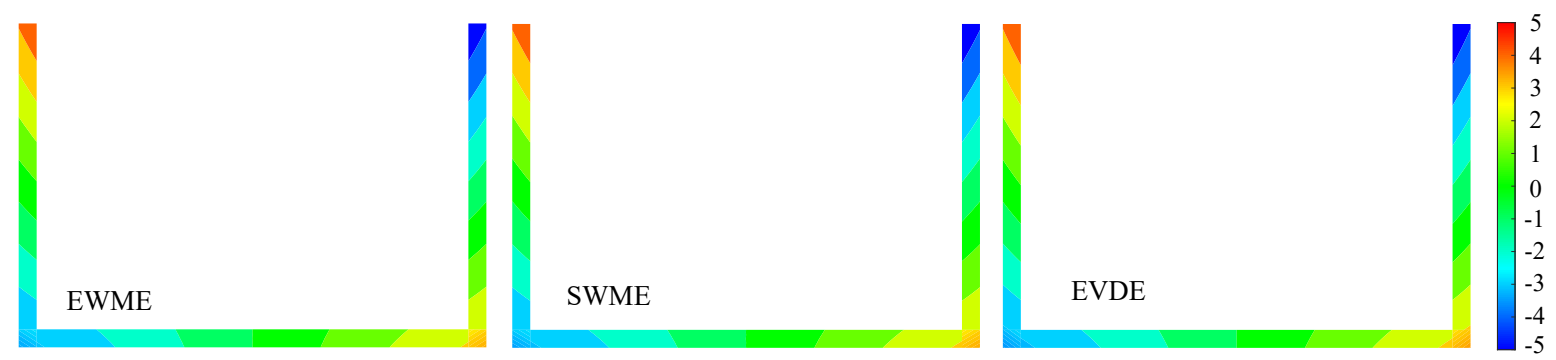

(a)

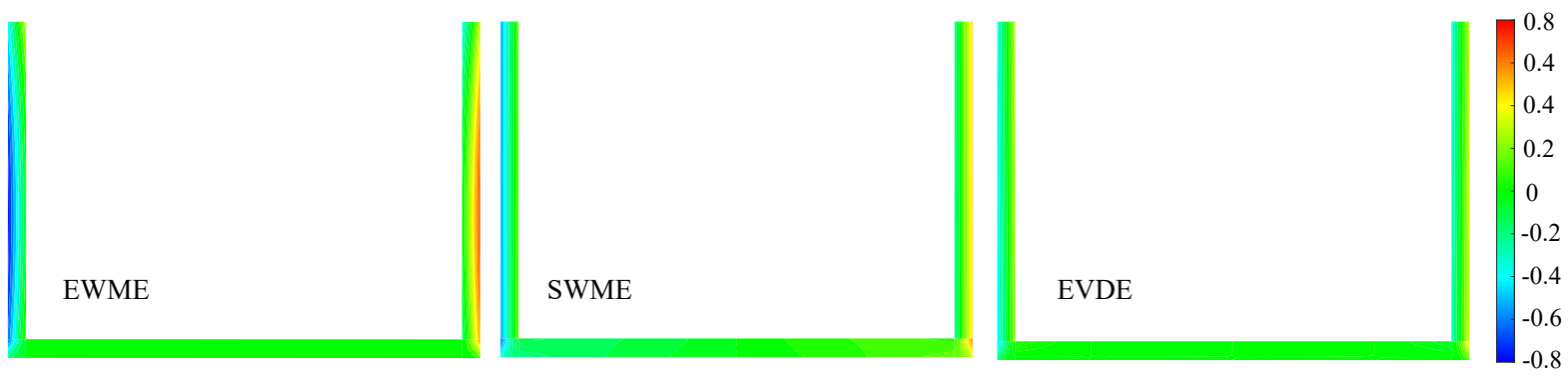

(b)

Figure 15: C-shaped cantilever: distribution of (a) axial and (b) tangential stresses, $\sigma_{x x}$ and $\tau_{x z}$, over the cross-section at $4.5 \mathrm{~m}$ from the fixed end, obtained with the EWME, SWME and EVDE (units MPa)

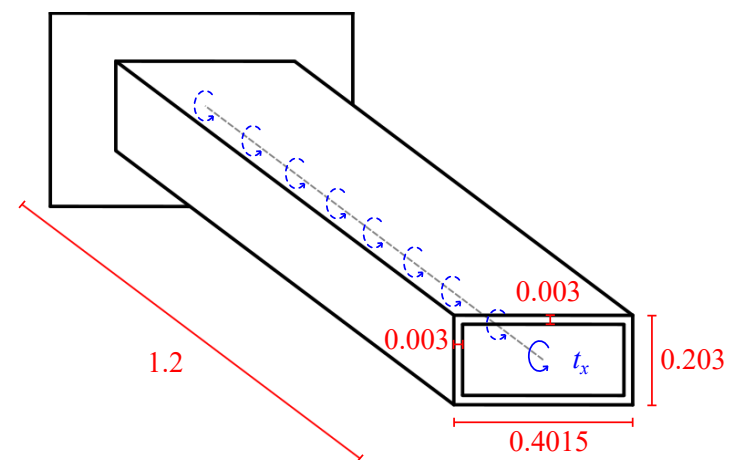

Figure 16: Boxed cantilever: static scheme and specimen geometry (all dimensions in meters) 
The beam is modeled by adopting a uniform mesh made of twelve FEs based on the proposed formulations. Test results are compared with those reported in [20], obtained by using two different FE approaches based on Vlasov's and Benscoter's theory [9, 10], respectively. For the SWME and EWME models, parabolic warping interpolation is assumed along the element axis, i.e. $n_{w}=3$. For the EWME, warping interpolation is assumed as cubic along web and flanges and quadratic across the membrature thickness, for a total number of warping DOFs at each cross-section equal to $m_{w}=62$.

Fig. 17 shows the variations along the element axis of the torsional rotation, $\theta_{x}$. As expected, EVDE (green curve with circles) and EWME (blue curve with dots) results perfectly agree with Vlasov's (dashed curve with crosses) and Benscoter's (dashed curve with square) solution, while intermediate results are given by the SWME model (red line with squares).

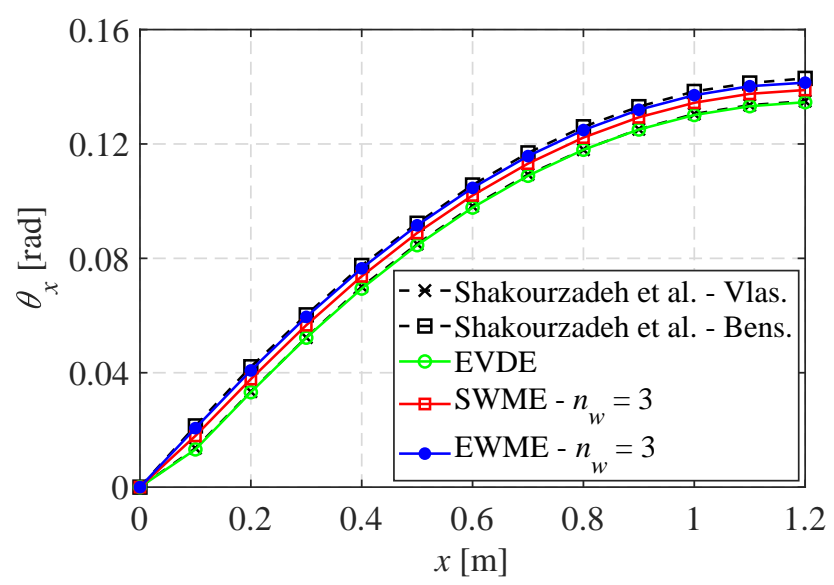

Figure 17: Boxed cantilever: axial variation of torsional rotation $\theta_{x}$

Similar trend is observed in Fig. 18, where the variation along the beam axis of (a) the warping parameter $\eta_{x}$ and (b) bimoment $B_{x}$ is reported (same curve styles of Fig. 17 are adopted). $\eta_{x}$ is null at the fixed end, where warping is restrained, and rapidly increases moving toward the free end. Opposite trend is observed for $B_{x}$, as warping restraints at the fixed end induce shear-lag effect that increases axial stresses, $\sigma_{x x}$, in this zone (see Eq. (32)). For the EWME, generalized warping quantities at the cross-section level are not available as well, as a detailed description of the warping is employed in this model at each integration point of the cross-section, according to the $m_{w}$ warping DOF scheme.

Finally, Figs. 19(a) and 19(b) plot the distributions of the axial and tangential stresses, $\sigma_{x x}$ and $\tau_{x z}$, respectively, evaluated with the three proposed models at $0.1 \mathrm{~m}$ from the fixed end, being $z$ the direction parallel to the flanges. As shown, EVDE provides distribution of the axial stress $\sigma_{x x}$ due to shear-lag effect that attains considerably lower values than those given by the EWME and SWME. However, no remarkable differences are observed for the $\tau_{x z}$ distribution. Indeed, as usual for closed profiles [21], shear stress exhibits a constant positive or negative flow through the thickness 


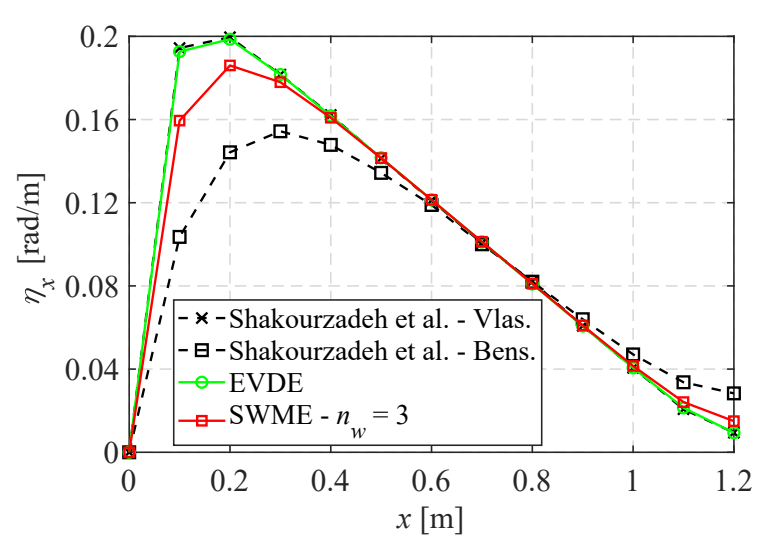

(a)

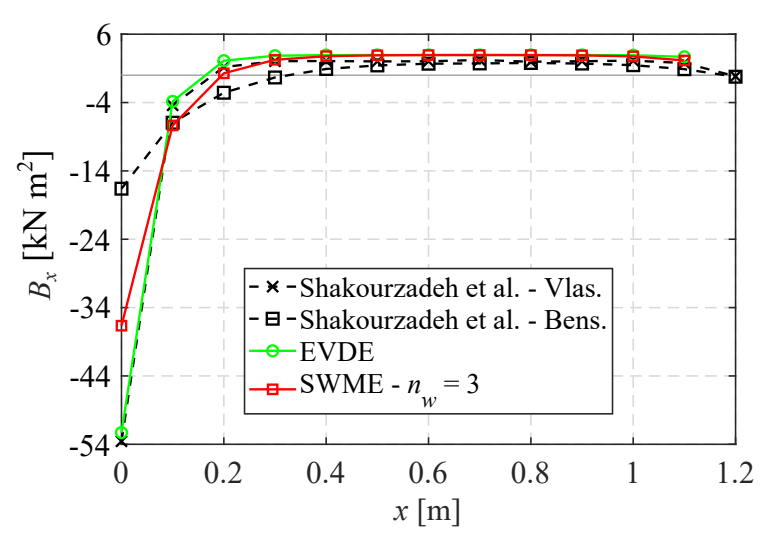

(b)

Figure 18: Boxed cantilever: axial variation of (a) warping parameter, $\eta_{x}$, and (b) bimoment, $B_{x}$

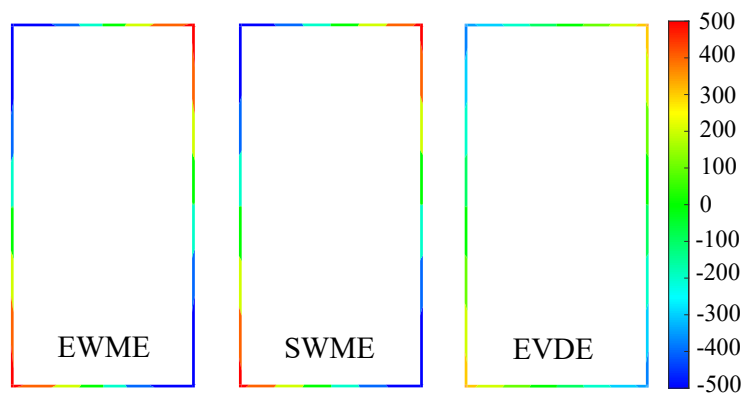

(a)
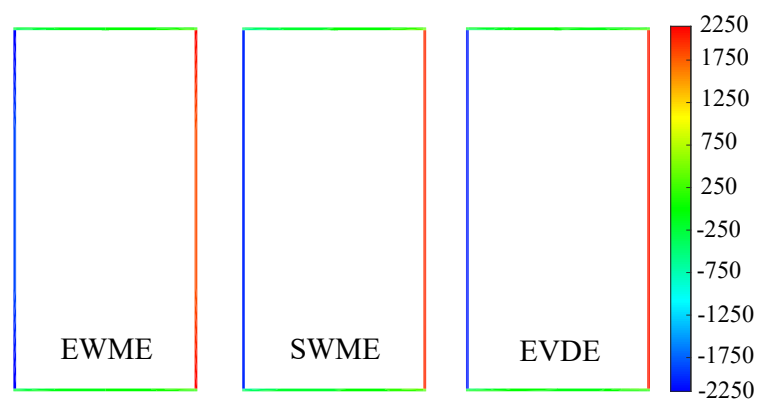

(b)

Figure 19: Boxed cantilever: distribution of (a) axial and (b) tangential stresses, $\sigma_{x x}$ and $\tau_{x z}$, over the cross-section at $0.1 \mathrm{~m}$ from the fixed end, obtained with the EWME, SWME and EVDE (units MPa)

of the membratures, that is shear stresses assume a regular variation in this direction that is well captured by all the proposed formulations, regardless of the assumed cross-section warping description.

\subsection{L frame with I-shaped members}

The fourth test is conducted to investigate the performances of the proposed formulations in analyzing a more complex structure composed by multiple members and study the interaction of warping deformation at the connection between beams and columns of a frame. Specimen geometry is depicted in Fig. 20(a). Rigid cross-section and warping displacements at the end of the beam (point A) and at the base of the column (point D) are prevented, as well as the transverse displacement along $z$ direction of the beam-column connection joint (point B). A concentrated torsional couple is applied at the column mid-height (point C), equal to $M_{x}=1.0 \mathrm{kNm}$. Two stiffener configurations are considered for the joint. These are depicted in Fig. 20(b) and indicated as diagonal and box/diagonal stiffened joint. Material behavior is defined by assuming Young's modulus, $E=205000 \mathrm{MPa}$ and Poisson ratio $v=0.3$. 


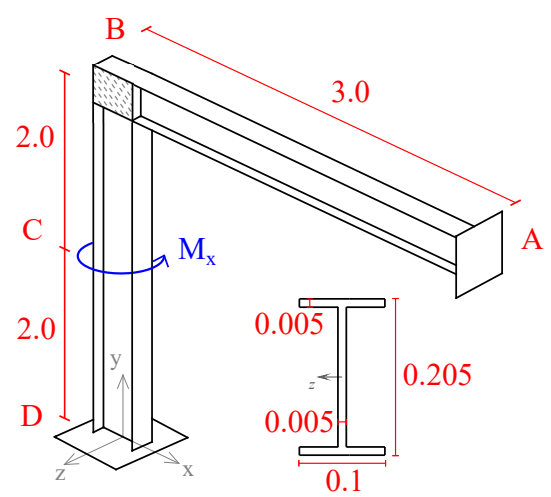

(a)

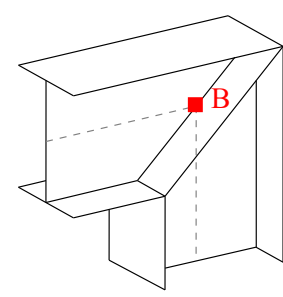

Diagonal stiffened

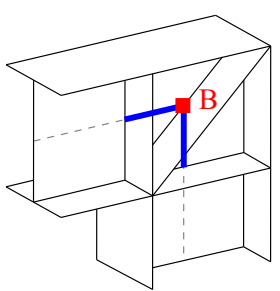

Box/diagonal stiffened

(b)

Figure 20: L frame: (a) static scheme and specimen geometry (all dimensions in meters) and (b) stiffener configurations at the joint

The frame is modeled by using the proposed beam formulations and adopting a uniform mesh made of eight FEs, for both beam and column. For the SWME and EWME models, parabolic warping interpolation is assumed along the element axis, i.e. $n_{w}=3$. For EWME model, parabolic and cubic warping interpolation is considered along web and flanges, respectively, and linear interpolation across the membrature thickness. The total number of warping DOFs over each cross-section is $m_{w}=28$. Warping boundary conditions at the joint are ensured by adopting the same modeling strategies adopted in [45], where similar frame is tested under dynamic loading conditions. For the box/diagonal stiffened joint, it is assumed that vertical and horizontal cross-sections, where the stiffeners are located, can not warp [6,58]. Hence, warping DOFs placed at these positions are restrained. For the EVDE, this is accomplished by restraining $\chi_{x}, \gamma_{y}$ and $\gamma_{z}$. Joint is modeled through rigid links that connect the beam to the column (blue lines in Fig. 20(b)), with a node of the mesh located at the link intersection, corresponding to point B. By contrast, for the diagonal stiffened joint, it is assumed that the end cross-sections of the members undergo equal warping profile. Hence, rigid links are not used and element axes meet at node B. In this case, for all models, mesh connectivity ensures that warping profile of the two cross-sections be the same, as both beam and column FEs connected at the joint share the same warping DOFs located at node B.

Fig. 21 shows the solution obtained for both diagonal and box/diagonal joint configurations in terms of torsional rotation $\theta_{x}$ of (a) beam and (b) column, referred to the element local axes $x$. The green circles, red squares and blue dots represent the results obtained with the EVDE, SWME and EWME models, respectively. These are compared with those obtained with the enhanced beam (solid black curves) and the shell (black triangles) model reported in [33]. Shell model is also used in [59] to test same specimen, providing similar results. Values of the rotation are reported assuming as positive direction for the element local axes that going from A to B for the beam and from D to B for the column. Torsion of the column mainly produces bending of the beam. However, for the diagonal stiffened joint, as warping of the column due to torsion induces warping in the beam, both elements undergo torsional deformations. By contrast, as warping transmission is prevented for the box/diagonal stiffened joint, in this case, cross-section torsional 


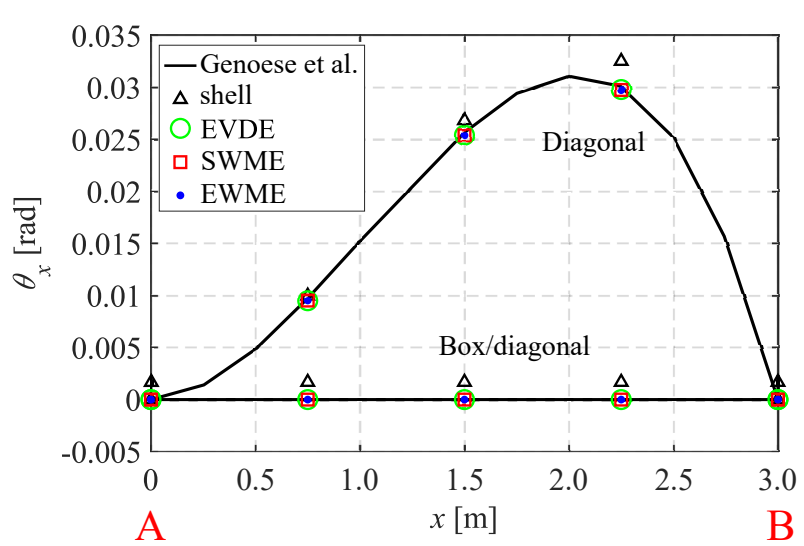

(a)

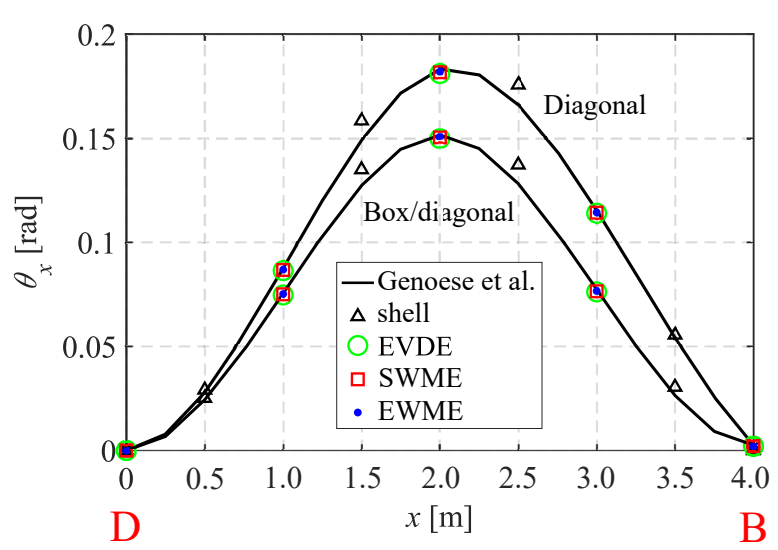

(b)

Figure 21: $\mathrm{L}$ frame: axial variation of torsional rotation, $\theta_{x}$, along local $x$-axis for (a) beam and (b) column

rotations are zero in the beam. As observed for the I-shaped cantilever in Section 4.2, all the proposed formulations give the same response that perfectly matches the beam solution in [33], for both joint configurations. As expected, the richer shell model provides slightly more flexible rotations, mostly at the member mid-spans.

\footnotetext{
9
} Fig. 22 shows the variation along the element local axis of the axial stress at the tip of the flanges for (a) beam and (b) column. The exact position of the monitored point $P$ is indicated by the sketches in the figure and the stresses is referred to as $\sigma_{x x}^{P}$. All proposed models provide same solution, reported with blue solid curves for the EWME, red dashed curves with stars for the SWME and green dashed curves for the EVDE. Comparison with shell model or other beam formulation is not reported in this case, as these data are not available in the reference papers.

In the column, axial stresses are mainly produced by shear-lag effect due to torsion and warping restraints at boundaries. By contrast, bending actions prevail in the beam. When the box/diagonal joint is considered, as torsional strains are zero in the beam, $\sigma_{x x}^{P}$ linearly varies along this element, while significant additional contribution associated to torsion arises in case of diagonal joint.

\section{Conclusions}

Three beam FE formulations for thin-walled structures were presented, based on the enrichment of the element kinematics description to include cross-section warping and correctly describe coupling between axial/bending and shear/torsional stress components. As opposed to the EWME model which introduces a detailed description of the warping displacement field at each point of the beam cross-section and was considered as the most sophisticated reference model, the EVDE and SWME formulations adopted a coarser approach based on a priori defined warping profiles. To be noted is that all the presented models were enriched to properly account for both the torsional and shear warping effects. In this perspective, the EVDE model can be considered as an enhancement of the classical Vlasov's formulation. At the same time, the SWME represents a simplified version of the more accurate EWME. The 


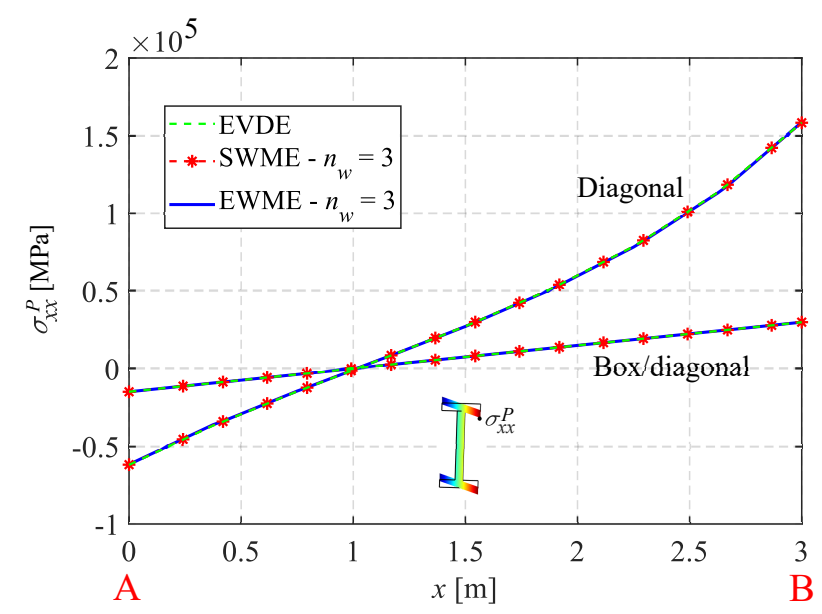

(a)

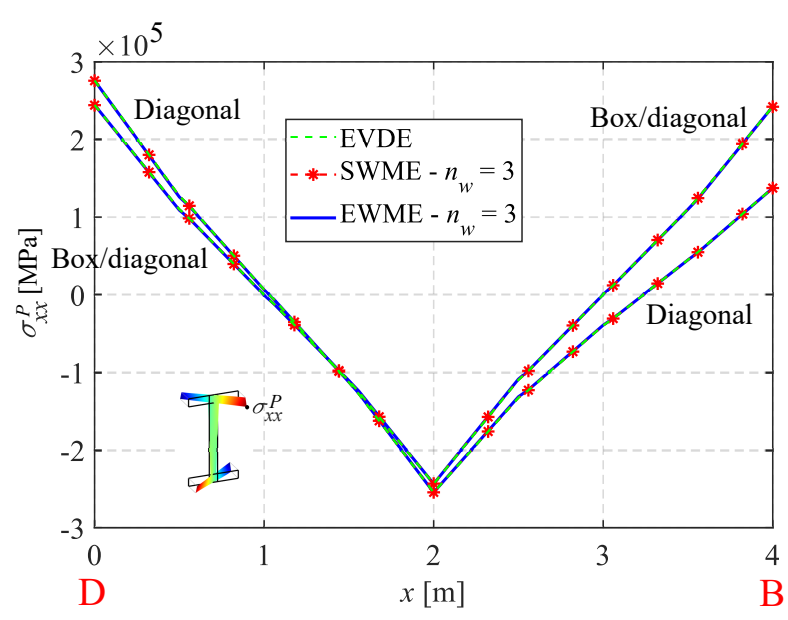

(b)

Figure 22: L frame: axial variation of axial stress, $\sigma_{x x}^{P}$, arising at the tip of the flanges for (a) beam and (b) column

investigation conducted on simple thin-walled structural elements showed that, as expected, EWME approach gives the most detailed results, in perfect agreement with the reference analytical/numerical solutions. However, this is the most computationally demanding, as it usually involves more additional warping DOFs than other formulations. On the other hand, the numerical examples proved that the other two EVDE and SWME models can be satisfactorily employed in various cases, mainly when the structural response does not involve complex warping deformation mechanisms. Indeed, the adoption of warping functions a priori defined over the element cross-sections limits the warping description provided by EVDE and SWME models, that, in some cases, result less accurate than EWME.

By adopting the EWME model, a numerical procedure was proposed to evaluate the warping profiles over the cross-sections, required for the EVDE and SWME. This is easily applicable to any cross-section geometry and proved to be a very efficient and extremely flexible technique, as it permits to choose the desired level of accuracy for the warping interpolation, similarly to the more sophisticated EWME model.

The response of four thin-walled specimens subjected to torsional loads was numerically reproduced, i.e. three cantilevers, with I-shaped, C-shaped and boxed cross-section, and a L frame with I-shaped members. The tests performed on the cantilever beams highlighted the performances of each proposed model in reproducing the response of symmetric, non-symmetric, opened and closed thin-walled profiles. These show that all formulations equally capture the behavior of thin-walled elements with opened doubly symmetric cross-sections. In fact, the influence of secondary shear stresses is negligible in this case and, thus, the coarser warping description adopted for the EVDE suffices to represent the element behavior. However, richer approaches are required for non-symmetric and/or closed profiles. The analyses on the $\mathrm{C}$-shaped and boxed cantilever showed that, although the global response of the beam was obtained by all the proposed models with sufficient accuracy, local variation of strains and stresses over the beam cross-sections and along the element axis was correctly capture only by more general formulations with independent 
warping parameters, as for SWME and EWME, which permit better estimation of the structural response. SWME model resulted a good compromise between the more complex and computational demanding EWME and the simpler, but less onerous, EVDE.

Convergence studies were also presented for the I-shaped and C-shaped cantilevers, to investigate the performances of the proposed formulations when different warping interpolation schemes are assumed along the element axis and over the cross-sections. As expected, the solution sensitivity to warping interpolation over the cross-section was observed only for thicker beams. By contrast, the shape function order assumed to describe warping variation along the element axis resulted crucial to improve the convergence speed and reduce the computational burden of the model.

The test performed on the $\mathrm{L}$ frame showed that the proposed beam formulations are also applicable for the analysis of full structures composed by multiple elements. Particular attention must be paid in the definition of the boundary conditions at the connection between beams and column. However, simple modeling strategies can be used to correctly reproduce the warping interaction occurring at element joints.

Lastly, from a computaional point of view, SWME and EWME approaches resulted both more performing than the EVDE, as these rely on more efficient FE mixed formulations, while the latter is based on the classical displacement approach. In fact, SWME and EWME models, based on mixed formulations, always showed faster convergence than EVDE. Hence, in spite of the lower computational cost requested by the single EVDE FE, finer mesh discretizations are usually required when this formulation is adopted.

\section{Acknowledgements}

D.A. and P.D.R. acknowledge the grant PRIN-2017 20173C478N.

\section{References}

[1] Gruttmann F, Sauer R, Wagner W. Shear stresses in prismatic beams with arbitrary cross-sections. International journal for numerical methods in engineering 1999;45(7):865-89.

[2] Pi YL, Trahair NS. Distortion and warping at beam supports. Journal of Structural Engineering 2000;126(11):1279-87.

[3] Montoya-Vargas S, Dario Aristizabal-Ochoa J. Torsion of prismatic I-beams with partially restrained warping at the end supports: Bending analogy method. Engineering Structures 2019;180:621-9. doi:10.1016/j.engstruct.2018.11.067.

[4] Jönsson J. Distortional warping functions and shear distributions in thin-walled beams. Thin-Walled Structures 1999;33(4):245-68.

[5] Pignataro M, Rizzi N, Ruta G, Varano V. The effects of warping constraints on the buckling of thin-walled structures. Journal of Mechanics of Materials and Structures 2009;4(10):1711-27.

[6] Rizzi NL, Varano V. The effects of warping on the postbuckling behaviour of thin-walled structures. Thin-Walled Structures 2011;49(9):1091-7.

[7] Dey P, Talukdar S. Influence of warping on modal parameters of thin-walled channel section steel beam. Procedia Engineering 2016;144:529.

[8] Rajkannu JS, Jayachandran SA. Flexural-torsional buckling strength of thin-walled channel sections with warping restraint. Journal of Constructional Steel Research 2020;169. 
[9] Vlasov VZ. Thin-walled elastic beams. National Technical Information Service; 1984.

[10] Benscoter S. A theory of torsion bending for multicell beams. Journal of Applied Mechanics 1954;21(1):25-34.

[11] Krajcinovic D. Matrix force analysis of thin-walled structures. Journal of the Structural Division 1970;96(1):107-21.

[12] Tralli AM. A simple hybrid model for torsion and flexure of thin-walled beams. Computers \& Structures 1986;22(4):649-58.

[13] Lee S, Kim Y. A new approach to the finite element modelling of beams with warping effect. International journal for numerical methods in engineering 1987;24(12):2327-41.

[14] Yoon K, Lee PS, Kim DN. An efficient warping model for elastoplastic torsional analysis of composite beams. Composite Structures 2017;178:37-49.

[15] Kim NI, Kim MY. Exact dynamic/static stiffness matrices of non-symmetric thin-walled beams considering coupled shear deformation effects. Thin-Walled Structures 2005;43(5):701-34.

[16] Dvorkin EN, Celentano D, Cuitino A, Gioia G. A Vlasov beam element. Computers \& structures 1989;33(1):187-96.

[17] Erkmen RE, Mohareb M. Torsion analysis of thin-walled beams including shear deformation effects. Thin-walled structures 2006;44(10):1096-108. doi:10.1016/j.tws.2006.10.012.

[18] Chen H, Blandford GE. A C0 finite element formulation for thin-walled beams. International Journal for Numerical Methods in Engineering 1989;28(10):2239-55.

[19] Back SY, Will KM. A shear-flexible element with warping for thin-walled open beams. International Journal for Numerical Methods in Engineering 1998;43(7):1173-91.

[20] Shakourzadeh H, Guo Y, Batoz JL. A torsion bending element for thin-walled beams with open and closed cross sections. Computers \& Structures 1995;55(6):1045-54.

[21] Saadé K, Espion B, Warzée G. Non-uniform torsional behavior and stability of thin-walled elastic beams with arbitrary cross sections. Thin-walled structures 2004;42(6):857-81.

[22] Dufort L, Grédiac M, Surrel Y. Experimental evidence of the cross-section warping in short composite beams under three point bending. Composite Structures 2001;51(1):37-47.

[23] Tsai HC, Kelly JM. Buckling of short beams with warping effect included. International Journal of Solids and Structures 2005;42(1):239-53.

[24] El Fatmi R. Non-uniform warping including the effects of torsion and shear forces. Part I: A general beam theory. International Journal of Solids and Structures 2007;44(18-19):5912-29.

[25] El Fatmi R. Non-uniform warping including the effects of torsion and shear forces. Part II: Analytical and numerical applications. International Journal of Solids and Structures 2007;44(18-19):5930-52.

[26] Alsafadie R, Hjiaj M, Battini JM. Three-dimensional formulation of a mixed corotational thin-walled beam element incorporating shear and warping deformation. Thin-Walled Structures 2011;49(4):523-33.

[27] Ruta G, Varano V, Pignataro M, Rizzi N. A beam model for the flexural-torsional buckling of thin-walled members with some applications. Thin-Walled Structures 2008;46(7-9):816-22.

[28] Rizzi NL, Varano V, Gabriele S. Initial postbuckling behavior of thin-walled frames under mode interaction. Thin-Walled Structures 2013;68:124-34.

[29] Camotim D, Basaglia C, Silvestre N. GBT buckling analysis of thin-walled steel frames: a state-of-the-art report. Thin-Walled Structures 2010;48(10-11):726-43.

[30] Vieira RF, Virtuoso FBE, Pereira EBR. A higher order thin-walled beam model including warping and shear modes. Int J Mech Sci 2013;66:67-82.

[31] Dikaros IC, Sapountzakis EJ. Generalized warping analysis of composite beams of an arbitrary cross section by BEM. I: Theoretical considerations and numerical implementation. J Eng Mech-ASCE 2014;140(9). doi:10.1061/(ASCE)EM.1943-7889.0000775.

[32] Capdevielle S, Grange S, Dufour F, Desprez C. A multifiber beam model coupling torsional warping and damage for reinforced concrete structures. Eur J Environ Civ En 2016;20:914-35.

[33] Genoese A, Genoese A, Bilotta A, Garcea G. A mixed beam model with non-uniform warping derived from the Saint Venànt rod. Computers 
\& Structures 2013;121:87-98.

[34] Genoese A, Genoese A, Bilotta A, Garcea G. A generalized model for heterogeneous and anisotropic beams including section distortions. Thin-Walled Structures 2014;74:85-103.

[35] Thomas D, Wilson J, Wilson R. Timoshenko beam finite elements. Journal of Sound and Vibration 1973;31(3):315-30.

[36] Hughes TJ, Taylor RL, Kanoknukulchai W. A simple and efficient finite element for plate bending. International Journal for Numerical Methods in Engineering 1977;11(10):1529-43.

[37] Tessler A, Dong S. On a hierarchy of conforming Timoshenko beam elements. Computers \& structures 1981;14(3-4):335-44.

[38] Zienkiewicz OC, Taylor RL. The finite element method for solid and structural mechanics. Elsevier; 2005.

[39] Caillerie D, Kotronis P, Cybulski R. A Timoshenko finite element straight beam with internal degrees of freedom. International Journal for Numerical and Analytical Methods in Geomechanics 2015;39(16):1753-73.

[40] Ciampi V, Carlesimo L. A nonlinear beam element for seismic analysis of structures. In: Proceedings of the 8th European Conference on Earthquake Engineering; vol. 3. 1986, p. 6-3.

[41] Spacone E, Ciampi V, Filippou F. Mixed formulation of nonlinear beam finite element. Computers \& Structures 1996;58(1):71-83.

[42] Addessi D, Di Re P. A 3D mixed frame element with multi-axial coupling for thin-walled structures with damage. Frattura ed Integrita Strutturale 2014;8(29):178-95.

[43] Di Re P, Addessi D, Filippou FC. Mixed 3D beam element with damage plasticity for the analysis of RC members under warping torsion. Journal of Structural Engineering 2018;144(6):04018064.

[44] Di Re P, Addessi D. A mixed 3D corotational beam with cross-section warping for the analysis of damaging structures under large displacements. Meccanica 2018;53(6):1313-32.

[45] Di Re P, Addessi D, Paolone A. Mixed beam formulation with cross-section warping for dynamic analysis of thin-walled structures. ThinWalled Structures 2019;141:554-75.

[46] Di Re P, Addessi D, Paolone A. Nonlinear dynamic analysis of thin-walled structures adopting a mixed beam finite element model with out-of-plane cross-section warping. vol. 2. Proceedings of the 7th ECCOMAS Thematic Conference on Computational Methods in Structural Dynamics and Earthquake Engineering (COMPDYN); 2019, p. 3611-32.

[47] Di Re P, Lofrano E, Addessi D, Paolone A. Enhanced beam formulation with cross-section warping under large displacements. vol. 2. Proceedings of the XXIV AIMETA Conference 2019. Lecture Notes in Mechanical Engineering; Springer; 2020 , p. $1217-29$.

[48] Saritas A, Filippou FC. Numerical integration of a class of $3 \mathrm{~d}$ plastic-damage concrete models and condensation of $3 \mathrm{~d}$ stress-strain relations for use in beam finite elements. Engineering Structures 2009;31(10):2327-36.

[49] Trahair NS. Nonlinear elastic nonuniform torsion. Journal of Structural Engineering 2005;131(7):1135-42.

[50] Taylor R, Filippou F, Saritas A, Auricchio F. A mixed finite element method for beam and frame problems. Computational mechanics 2003;31(1-2):192-203.

[51] Timoshenko SP, Gere JM. Theory of elastic stability. Courier Corporation; 2009.

[52] Le Corvec V. Nonlinear 3d frame element with multi-axial coupling under consideration of local effects. 2012. Ph.D thesis, University of California, Berkeley.

[53] Di Re P. 3D beam-column finite elements under tri-axial stress-strain states: nonuniform shear stress distribution and warping. 2017. Ph.D thesis, Sapienza University of Rome; URL http://hdl . handle.net/11573/937922.

[54] Di Re P, Addessi D, Filippou FC. 3D beam-column finite element under non-uniform shear stress distribution due to shear and torsion. In: Proceedings of the VII ECCOMAS Congress; vol. 3. 2016, p. 4467-80.

[55] Kostic SM, Filippou FC. Section discretization of fiber beam-column elements for cyclic inelastic response. Journal of Structural Engineering 2012;138(5):592-601.

[56] Wilson EL. Three dimensional static and dynamic analysis of structures: a physical approach with emphasis on earthquake engineering. Computers and structures; 2002.

[57] Yoon K, Lee Y, Lee PS. A continuum mechanics based 3-d beam finite element with warping displacements and its modeling capabilities. 
Structural Engineering and Mechanics 2012;43(4):411-37.

[58] Basaglia C, Camotim D, Silvestre N. Global buckling analysis of plane and space thin-walled frames in the context of GBT. Thin-Walled Structures 2008;46(1):79-101.

812 [59] Basaglia C, Camotim D, Silvestre N. Torsion warping transmission at thin-walled frame joints: Kinematics, modelling and structural response.

Journal of Constructional Steel Research 2012;69(1):39-53. 